\begin{tabular}{lll} 
Universidade do Minho & Universidade do Minho \\
Escola de Ciências & $\begin{array}{l}\text { Universidade do Minho } \\
\text { Escola de Ciências }\end{array}$ \\
Centro de Matemática & Departamento de Matemática e Aplicações \\
\hline
\end{tabular}

\title{
Iteration of Quadratic Maps on Coquaternions
}

\author{
M. Irene Falcão ${ }^{a, c} \quad$ Fernando Miranda $^{a, c} \quad$ Ricardo Severino $^{c} \quad$ M. Joana Soares ${ }^{b, c}$
}

a CMAT - Centre of Mathematics, University of Minho, Portugal

${ }^{b}$ NIPE - Economic Policies Research Unit, University of Minho, Portugal

${ }^{c}$ Department of Mathematics and Applications, University of Minho, Portugal

\section{Information}

Keywords:

Iteration of quadratic maps, fixed points, periodic points, bifurcations coquaternions.

Original publication: International Journal of Bifurcation and Chaos Vol. 27, No. 12 (2017) 1730039 (22 pages)

DOI:10.1142/S0218127417300397

World Scientific Publishing Company

\begin{abstract}
This paper is concerned with the study of the iteration of the quadratic coquaternionic map $f_{c}(q)=q^{2}+c$, where $c$ is a fixed coquaternionic parameter. The fixed points and periodic points of period two are determined, revealing the existence of a type of sets of these points which do not occur in the classical complex case: sets of non-isolated points. This brings the need to consider a different concept of stability. The analysis of the stability, in this new sense, of the sets of fixed points and periodic points is performed and a discussion of certain type of bifurcations which occur, in the case of a real parameter $\mathrm{c}$, is also presented.
\end{abstract}

\section{Introduction}

When seeking to extend some area of mathematics, developed under a specific context, to a more general setting, one is usually faced with two confronting issues: the first is the natural expectation that new results and phenomena will emerge; the second is the possible failure, in the wider framework, of techniques that have proven to be extremely effective in the first context and which were essential to the understanding of the mathematics there involved.

Quaternions are usually considered as the natural generalization of complex numbers. Hence, it is not surprising that the first attempts of extending the well-established iteration theory of quadratic maps in the complex plane to higher dimensions involved quaternionic maps; see e.g. $[5,6,8,12,13,16]$. It turns out that this generalization proved not to be very fruitful, with the new results being very closely related to the corresponding ones in the complex case; for example, as proved by Nakane [16], the only attractive periodic cycles of quadratic quaternionic maps can be reduced to complex attractive periodic cycles. However, quaternions are not the only four dimensional hypercomplex real algebra generalizing complex numbers; for example, one may also consider coquaternions, introduced by Sir James Cockle (1819-1895) at about the same time as Sir William Hamilton (1805-1865) discovered the quaternions; [9].

This paper is devoted to the study of the iteration of the quadratic coquaternionic map $f_{c}(q)=q^{2}+c$, where $\mathrm{c}$ is a fixed coquaternionic parameter, with particular interest in the determination and subsequent stability analysis of its fixed points and periodic points of period two. 
We should observe that, since the algebra of coquaternions is isomorphic to the algebra $M_{2}(\mathbb{R})$ of $2 \times 2$ real matrices - see, e.g. [14] —, the research conducted, in this matricial framework, by Baptista et al. [4] and Serenevy [18] can be seen as a first important contribution to the study of the dynamics of coquaternionic maps, already revealing an unexpected richness of results when compared with the quaternionic case. Here, however, we do not make use of matrices, but employ a coquaternionic formalism. In our opinion, the use of this formalism, besides making the presentation more elegant, greatly facilitates the obtainment and interpretation of results. We not only rediscover some of the results present in the aforementioned papers by Baptista et al. [4] and Serenevy [18], but also find new interesting results not contained in these references. For example, even in the simplest case of a parameter $c \in \mathbb{R}-$ a case not studied by the referred authors - we encounter a type of sets of fixed points and of periodic points of period two which do not occur in the classical complex case: sets of non-isolated points. This situation brings the need to modify the usual concept of attractive periodic point - adequate in the case of an isolated point - replacing it by the notion of attractive set of periodic points. With this new concept of stability, we find (again, even when we restrict to the case of a real parameter) that there are maps which admit two distinct attractive sets of periodic points of period two: one, corresponding to the classical case, formed by two isolated points, and another which is a made up of non-isolated points.

The rest of the paper proceeds as follows: Section 2 contains a summary of the basic results on the algebra of coquaternions and in Section 3 we give some definitions and preliminary results on the coquaternionic quadratic map $f_{c}$; Section 4 is dedicated to the determination of the fixed points and periodic points of period two of $f_{c}$, whose stability is then analyzed in Section 5; in Section 6 we discuss the existence, for the parameter $c \in \mathbb{R}$, of phenomena which can be seen as a kind of period-doubling and pitchfork bifurcations for sets of non-isolated points; finally, Section 7 concludes.

\section{Some Basic Results on Coquaternions}

The algebra of real coquaternions, ${ }^{1}$ which we will denote by $\mathbb{H}_{\text {coq }}$, is obtained by endowing the vector space $\mathbb{R}^{4}$ with the following product: if $\{1, \mathbf{i}, \mathbf{j}, \mathbf{k}\}$ denotes the standard basis, define

$$
\begin{gathered}
\mathbf{i}^{2}=-1, \mathbf{j}^{2}=\mathbf{k}^{2}=1, \\
\mathbf{i j}=-\mathbf{j i}=\mathbf{k}, \mathbf{k j}=-\mathbf{j} \mathbf{k}=\mathbf{i}, \mathbf{k} \mathbf{i}=-\mathbf{i} \mathbf{k}=\mathbf{j},
\end{gathered}
$$

let 1 be the neutral element and extend the operation by distributivity to all coquaternions $\mathrm{q}=q_{0}+q_{1} \mathbf{i}+$ $q_{2} \mathbf{j}+q_{3} \mathbf{k}$. This is an associative and non-commutative algebra. It is also important to observe that, contrary to what happens in the case of Hamiltonian quaternions, $\mathbb{H}_{\text {coq }}$ is not a division algebra. In fact, $\mathbb{H}_{\text {coq }}$ contains zero divisors and nilpotent elements. For example, in $\mathbb{H}_{\text {coq }}$, we have $(1+\mathbf{j})(1-\mathbf{j})=0$ and $(\mathbf{i}+\mathbf{j})^{2}=0$.

The explicit multiplication of two coquaternions $\mathrm{p}=p_{0}+p_{1} \mathbf{i}+p_{2} \mathbf{j}+p_{3} \mathbf{k}$ and $q=q_{0}+q_{1} \mathbf{i}+q_{2} \mathbf{j}+q_{3} \mathbf{k}$ is given by

$$
\begin{aligned}
\mathrm{pq}=p_{0} q_{0}-p_{1} q_{1}+p_{2} q_{2}+p_{3} q_{3}+\left(p_{0} q_{1}\right. & \left.+p_{1} q_{0}-p_{2} q_{3}+p_{3} q_{2}\right) \mathbf{i} \\
& +\left(p_{0} q_{2}-p_{1} q_{3}+p_{2} q_{0}+p_{3} q_{1}\right) \mathbf{j}+\left(p_{0} q_{3}+p_{1} q_{2}-p_{2} q_{1}+p_{3} q_{0}\right) \mathbf{k}
\end{aligned}
$$

from where we immediately obtain

$$
\mathrm{q}^{2}=q_{0}^{2}-q_{1}^{2}+q_{2}^{2}+q_{3}^{2}+2 q_{0}\left(q_{1} \mathbf{i}+q_{2} \mathbf{j}+q_{3} \mathbf{k}\right) .
$$

Given a coquaternion $\mathrm{q}=q_{0}+q_{1} \mathbf{i}+q_{2} \mathbf{j}+q_{3} \mathbf{k} \in \mathbb{H}_{\text {coq }}$, its conjugate $\overline{\mathrm{q}}$ is defined as $\overline{\mathrm{q}}=q_{0}-q_{1} \mathbf{i}-q_{2} \mathbf{j}-q_{3} \mathbf{k}$; the number $q_{0}$ is called the real part of $q$ and denoted by re $q$ and the vector part of $q$, denoted by vec $q$, is given by vec $\mathrm{q}=q_{1} \mathbf{i}+q_{2} \mathbf{j}+q_{3} \mathbf{k}$. We will denote by $\operatorname{tr} \mathrm{q}$ and call trace of $\mathrm{q}$ the quantity given by $\operatorname{tr} \mathrm{q}=\mathrm{q}+\overline{\mathrm{q}}=2$ re $\mathrm{q}$ and call determinant of $\mathrm{q}$, denoted by $\operatorname{det} \mathrm{q}$, to the quantity given by

$$
\operatorname{det} \mathrm{q}=\mathrm{q} \overline{\mathrm{q}}=q_{0}^{2}+q_{1}^{2}-q_{2}^{2}-q_{3}^{2} .
$$

We will identify the set of coquaternions whose vector part is zero with the set $\mathbb{R}$ of real numbers and the set of coquaternions such that $q_{2}=q_{3}=0$ with the set $\mathbb{C}$ of complex numbers.

The following proposition lists some properties of coquaternions which are easily verified; see e.g. [1].

\footnotetext{
${ }^{1}$ Also known, in the literature, as split-quaternions, para-quaternions, anti-quaternions or hyperbolic quaternions.
} 
Proposition 2.1. Given $\mathrm{p}, \mathrm{q} \in \mathbb{H}_{\mathrm{coq}}$ and $\alpha \in \mathbb{R}$, we have:

(i) $\overline{\mathrm{p}+\mathrm{q}}=\overline{\mathrm{p}}+\overline{\mathrm{q}}$

(ii) $\overline{\mathrm{pq}}=\overline{\mathrm{q}} \overline{\mathrm{p}}$

(iii) $\operatorname{re}(\mathrm{pq})=\operatorname{re}(\mathrm{qp})$

(iv) $\operatorname{det} q=\operatorname{det} \bar{q}$

(v) $\operatorname{det}(\alpha \mathrm{q})=\alpha^{2} \operatorname{det} \mathrm{q}$

(vi) $\operatorname{det}(p q)=\operatorname{det}(q p)=\operatorname{det} p \operatorname{det} q$

(vii) q commutes with any other coquaternion if and only if $\mathrm{q} \in \mathbb{R}$ (i.e. the center of $\mathbb{H}_{\mathrm{coq}}$ is $\mathbb{R}$ ).

Contrary to what happens in the case of quaternions, not all non-zero coquaternions are invertible. The following proposition characterizes the invertible coquaternions.

Proposition 2.2. A coquaternion $\mathrm{q}$ is invertible if and only if $\operatorname{det} \mathrm{q} \neq 0$. In that case, we have $\mathrm{q}^{-1}=\frac{\overline{\mathrm{q}}}{\operatorname{det} \mathrm{q}}$.

Proof. The sufficient condition is proved by simple verification; the necessary condition is a simple consequence of the result (vi) in Proposition 2.1, by observing that $\operatorname{det} 1=1$.

We say that a coquaternion $\mathrm{q}$ is similar to a coquaternion $\mathrm{p}$, and write $\mathrm{q} \sim \mathrm{p}$, if there exists an invertible coquaternion h such that $\mathrm{p}=\mathrm{hqh}^{-1}$. This is an equivalence relation in $\mathbb{H}_{\text {coq }}$, partitioning $\mathbb{H}_{\text {coq }}$ in the so-called similarity classes. We denote by $[\mathrm{q}]$ the similarity class containing the coquaternion $\mathrm{q}$. One can easily show that $[\mathrm{q}]=\{\mathrm{q}\}$ if and only if $\mathrm{q}$ is real.

For an invertible coquaternion $\mathrm{h}$, let $\phi_{\mathrm{h}}: \mathbb{H}_{\mathrm{coq}} \rightarrow \mathbb{H}_{\mathrm{coq}}$ be the associated similarity map defined by

$$
\phi_{\mathrm{h}}(\mathrm{q})=\mathrm{h}^{-1} \mathrm{qh} \text {. }
$$

It is simple to verify that $\phi_{\mathrm{h}}$ is an algebra automorphism for $\mathbb{H}_{\text {coq }}$.

It is a well-known result that any Hamilton quaternion is similar to a complex number with non-negative real part - see e.g. [7, Lemma 3] - thus allowing the choice of that special form for the representative of any similarity class. The following result shows that the situation is different in the case of coquaternions, where three different types of representatives need to be used (for non-real coquaternions).

Theorem 2.1. Let $\mathrm{q}=q_{0}+q_{1} \mathbf{i}+q_{2} \mathbf{j}+q_{3} \mathbf{k}=q_{0}+$ vec $\mathrm{q}$ be a non-real coquaternion and let

$$
r=\operatorname{det}(\operatorname{vec} \mathrm{q})=q_{1}^{2}-q_{2}^{2}-q_{3}^{2} .
$$

(a) If $r>0$, there exists a similarity transformation $\phi_{\mathrm{h}}$ such that $\phi_{\mathrm{h}}(\mathrm{q})=q_{0}+\sqrt{r} \mathbf{i}$.

(b) If $r<0$, there exists a similarity transformation $\phi_{\mathrm{h}}$ such that $\phi_{\mathrm{h}}(\mathrm{q})=q_{0}+\sqrt{-r} \mathbf{j}$.

(c) If $r=0$, there exists a similarity transformation $\phi_{\mathrm{h}}$ such that $\phi_{\mathrm{h}}(\mathrm{q})=q_{0}+\mathbf{i}+\mathbf{j}$.

Proof. The proof of the results (a) and (b) can be see in [15]; to establish (c), simply note that we can take $\mathrm{h}=\left(1+q_{1}\right)-q_{3} \mathbf{j}-\left(1-q_{2}\right) \mathbf{k}$, if $q_{1}+q_{2} \neq 0$, and $\mathrm{h}=\left(1+q_{1}\right) \mathbf{i}+\left(1-q_{1}\right) \mathbf{j}$, otherwise.

Proposition 2.3. Two non-real coquaternions $\mathrm{p}$ and $\mathrm{q}$ are similar if and only if

$$
\operatorname{rep}=\operatorname{req} \text { and } \operatorname{det}(\operatorname{vec} \mathrm{p})=\operatorname{det}(\operatorname{vec} \mathrm{q}) \text {. }
$$

Proof. If two coquaternions are similar, then it is an immediate consequence of ( $v$ ) and (vi) in Proposition 2.1 and the fact that, for any invertible coquaternion $h$, we have $\operatorname{det}\left(\mathrm{h}^{-1}\right)=\frac{1}{\operatorname{det} \mathrm{h}}$, that they must satisfy the above conditions. The fact that two non-real coquaternions p and q satisfying (4) are similar is an immediate consequence of the previous theorem, having in mind that similarity is an equivalence relation.

Remark 2.1. We should emphasize that, since a real coquaternion is never similar to any other coquaternion, the conditions (4) guarantee the similarity of $\mathrm{p}$ and $\mathrm{q}$ only if both these coquaternions are non-real. 
Remark 2.2. We can identify the similarity class of any non-real coquaternion q with an hyperboloid in the hyperplane $\left\{\left(x_{0}, x_{1}, x_{2}, x_{3}\right) \in \mathbb{R}^{4}: x_{0}=q_{0}\right\}$ : this will be an hyperboloid of two sheets, an hyperboloid of one sheet or a degenerate hyperboloid, i.e. a cone, depending on whether $\operatorname{det}(\operatorname{vec} \mathrm{q})>0, \operatorname{det}(\operatorname{vec} \mathrm{q})<0$ or $\operatorname{det}(\operatorname{vec} \mathrm{q})=0$, respectively. ${ }^{2}$

\section{Coquaternionic Quadratic Map}

Our main interest is to study the dynamics of the quadratic map

$$
\begin{aligned}
f_{\mathrm{c}}: \mathbb{H}_{\mathrm{coq}} & \rightarrow \mathbb{H}_{\text {coq }} \\
\mathrm{q} & \mapsto \mathrm{q}^{2}+\mathrm{c}
\end{aligned}
$$

where $\mathrm{c}$ is a fixed parameter in $\mathbb{H}_{\text {coq }}$.

\subsection{Preliminary results}

We first recall several basic definitions and present some results which will play an important role in the remaining part of the paper. For $k \in \mathbb{N}$, we shall denote by $f_{\mathrm{c}}^{k}$ the $k$-th iterate of $f_{\mathrm{c}}$, inductively defined by $f_{\mathrm{c}}^{0}=\mathrm{id}_{\mathbb{H}_{\mathrm{coq}}}$ and $f_{\mathrm{c}}^{k}=f_{\mathrm{c}} \circ f_{\mathrm{c}}^{k-1}$. For a given initial point $\mathrm{q} \in \mathbb{H}_{\mathrm{coq}}$, the orbit of $\mathrm{q}$ under $f_{\mathrm{c}}$ is the set $\mathcal{O}(\mathrm{q}):=\left\{f_{\mathrm{c}}^{k}(\mathrm{q})\right\}_{k \in \mathbb{N}_{0}}$. The point $\mathrm{q}$ is said to be a periodic point of $f_{\mathrm{c}}$ with period $n \in \mathbb{N}$, if $f_{\mathrm{c}}^{n}(\mathrm{q})=\mathrm{q}$, with $f_{\mathrm{c}}^{k}(\mathrm{q}) \neq \mathrm{q}$ for $0<k<n$; in this case, the set $\mathcal{O}(\mathrm{q})$ is called an $n$-cycle for $f_{\mathrm{c}}$. If $\mathrm{q}$ is a periodic point of period 1 , i.e. if $f_{\mathrm{c}}(\mathrm{q})=\mathrm{q}$, we say that $\mathrm{q}$ is a fixed point for $f_{\mathrm{c}}$.

The important result contained in the following lemma can be shown by a simple verification.

Lemma 3.1. For any coquaternion $\mathrm{q} \in \mathbb{H}_{\mathrm{coq}}$, we have

$$
q^{2}=(\operatorname{tr} q) q-\operatorname{det} q
$$

The next theorem establishes that the orbit of any coquaternion q lies in a subspace of $\mathbb{H}_{\text {coq }}$ whose dimension is, at most, equal to three.

Theorem 3.1. Let $f_{\mathrm{c}}$ be the quadratic map defined by (5). Then, for any initial $\mathrm{q} \in \mathbb{H}_{\mathrm{coq}}$, the orbit of $\mathrm{q}$ lies in the subspace $\operatorname{span}_{\mathbb{R}}(1, \mathrm{c}, \mathrm{q})$. Furthermore:

- if $\mathrm{c} \in \mathbb{R}$, the orbit of $\mathrm{q}$ lies in the subspace $\operatorname{span}_{\mathbb{R}}(1, \mathrm{q})$;

- if $\mathrm{q} \in \operatorname{span}_{\mathbb{R}}(1, \mathrm{c})$, the orbit of $\mathrm{q}$ lies in the subspace $\operatorname{span}_{\mathbb{R}}(1, \mathrm{c})$.

Proof. The first assertion follows easily from Lemma 3.1, by observing that, for any $q \in \mathbb{H}_{\text {coq }}$,

$$
f_{c}(\mathrm{q})=\mathrm{q}^{2}+\mathrm{c}=\alpha \mathrm{q}+\beta \mathrm{c}+\gamma
$$

with $\alpha=\operatorname{tr} q, \beta=1$ and $\gamma=-\operatorname{det} q$. Hence, $f_{\mathrm{c}}(\mathrm{q}) \in \operatorname{span}_{\mathbb{R}}(1, \mathrm{c}, \mathrm{q})$ and the same is true for to all the subsequent iterates. The other results follow by noting that, if $c \in \mathbb{R}$, then $\operatorname{span}_{\mathbb{R}}(1, \mathrm{c}, \mathrm{q})=\operatorname{span}_{\mathbb{R}}(1, \mathrm{q})$ and if $\mathrm{q} \in \operatorname{span}_{\mathbb{R}}(1, \mathrm{c})$, then $\operatorname{span}_{\mathbb{R}}(1, \mathrm{c}, \mathrm{q})=\operatorname{span}_{\mathbb{R}}(1, \mathrm{c})$.

Lemma 3.2. Let $\phi_{\mathrm{h}}$ be the similarity map defined by (3) for a given invertible coquaternion $\mathrm{h}$. The dynamical system $\left(\mathbb{H}_{\mathrm{coq}}, f_{\mathrm{c}}\right)$ is dynamically equivalent to the dynamical system $\left(\mathbb{H}_{\mathrm{coq}}, f_{\phi_{\mathrm{h}}(\mathrm{c})}\right)$.

Proof. We have

$$
\begin{aligned}
\left(\phi_{\mathrm{h}} \circ f_{\mathrm{c}} \circ \phi_{\mathrm{h}}^{-1}\right)(\mathrm{q})=\left(\phi_{\mathrm{h}} \circ f_{\mathrm{c}}\right)\left(\mathrm{hqh}^{-1}\right) & =\phi_{\mathrm{h}}\left(\left(\mathrm{hqh}^{-1}\right)^{2}+\mathrm{c}\right) \\
= & \phi_{\mathrm{h}}\left(\mathrm{hq}^{2} \mathrm{~h}^{-1}+\mathrm{c}\right)=\mathrm{h}^{-1}\left(\mathrm{hq}^{2} \mathrm{~h}^{-1}+\mathrm{c}\right) \mathrm{h}=\mathrm{q}^{2}+\mathrm{h}^{-1} \mathrm{ch}=f_{\phi_{\mathrm{h}}(\mathrm{c})}(\mathrm{q})
\end{aligned}
$$

\footnotetext{
${ }^{2}$ Strictly speaking, we have to exclude the point $\left(q_{0}, 0,0,0\right)$ of the cone from the equivalence class in case $\operatorname{det}(\operatorname{vec} \mathrm{q})=0$.
} 
As a consequence of Lemma 3.2 above and of Theorem 2.1, we immediately obtain the result contained in the following theorem.

Theorem 3.2. To study the dynamics of the quadratic map $f_{\mathrm{c}}(\mathrm{q})=\mathrm{q}^{2}+\mathrm{c}$, there is no loss of generality in assuming that $\mathrm{c}$ has one of the following forms, where $c_{0}, c_{1}, c_{2} \in \mathbb{R}$ :

$$
\begin{array}{ll}
\mathcal{C}_{0} & \mathrm{c}=c_{0} \\
\mathcal{C}_{1} & \mathrm{c}=c_{0}+c_{1} \mathbf{i}, \quad c_{1}>0 \\
\mathcal{C}_{2} & \mathrm{c}=c_{0}+c_{2} \mathbf{j}, \quad c_{2}>0 \\
\mathcal{C}_{3} & \mathrm{c}=c_{0}+\mathbf{i}+\mathbf{j}
\end{array}
$$

We will refer to the four different forms of the parameter c given above as the canonical forms.

\subsection{Dynamics in the canonical planes}

We consider now three particularly important subspaces of dimension two of $\mathbb{H}_{\text {coq }}$, usually called the canonical planes or cycle planes. The first is simply the complex plane $\mathbb{C}$; the second, which we denote by $\mathbb{P}$ and whose elements are usually called perplex numbers ${ }^{3}$ is given by $\mathbb{P}=\operatorname{span}_{\mathbb{R}}(1, \mathbf{j})$ and corresponds to the classical Minkowski plane; the third, denoted by $\mathbb{D}$, is the subspace of the so-called dual numbers, $\mathbb{D}=\operatorname{span}_{\mathbb{R}}(1, \mathbf{i}+\mathbf{j})$ and can be identified with the classical Laguerre plane. Note that $\mathrm{c}=c_{0} \in \mathbb{R}$ belongs to any of these planes and, if $\mathrm{c}$ has the canonical form $\mathcal{C}_{1}$, then $\mathrm{c} \in \mathbb{C}$, if $\mathrm{c}$ has the canonical form $\mathcal{C}_{2}$, then $\mathrm{c} \in \mathbb{P}$ and if $\mathrm{c}$ has the canonical form $\mathcal{C}_{3}$, then $\mathrm{c} \in \mathbb{D}$.

The dynamics of the quadratic map in the complex plane has been object of intense study. More recently, the study of the dynamics of this map restricted to the other two cycle planes has also drawn the attention of several authors; see, e.g. [2, 10, 11, 17]; a discussion based on the use of $2 \times 2$ matrices can also be seen in [18]. In the rest of this section we present a very brief discussion of the results on the dynamics of $f_{\mathrm{c}}$ in each of the cycle planes which are relevant to our future work.

\subsubsection{Dynamics in $\mathbb{C}$}

We first recall the well-known expressions for the fixed points and periodic points of period two of the complex map $f_{\mathrm{c}}$. We have the following fixed points

$$
x_{ \pm}=\frac{1}{2}(1 \pm \sqrt{1-4 \mathrm{c}})
$$

and the following periodic points of period two

$$
y_{ \pm}=-\frac{1}{2}(1 \pm \sqrt{-3-4 \mathrm{c}}), \quad \mathrm{c} \neq-\frac{3}{4} .
$$

Note that when $\mathrm{c}=c_{0} \in \mathbb{R}$, the points given by (7) are real for $c_{0} \leq \frac{1}{4}$ and complex conjugate for $c_{0}>\frac{1}{4}$ and the points given by (8) are real for $c_{0}<-3 / 4$ and complex conjugate for $c_{0}>-3 / 4$. In this case of a real parameter $\mathrm{c}=c_{0}$, we have that $x_{-}$is attractive for $-3 / 4<c_{0}<1 / 4$ and repelling for $c_{0}<-3 / 4$ or $c_{0}>1 / 4$, whilst the point $x_{+}$is always repelling (except when $c_{0}=\frac{1}{4}$, in which case we have the neutral point $x=\frac{1}{2}$ ). The 2 -cycle defined by (8) is attractive for $-5 / 4<c_{0}<-3 / 4$ and repelling for $c_{0}<-5 / 4$ or $c_{0}>-3 / 4$. For $c_{0}=-5 / 4$, the 2 -cycle is neutral.

\subsubsection{Dynamics in $\mathbb{P}$}

Consider the case where $\mathrm{c}$ and $\mathrm{q}$ both belong to the plane $\mathbb{P}$, i.e. suppose that $\mathrm{q}=q_{0}+q_{2} \mathbf{j}$ and $\mathrm{c}=c_{0}+c_{2} \mathbf{j}$. We can use the so-called dual basis of $\mathbb{P}$ formed by the two elements $e_{1}=\frac{1}{2}(1+\mathbf{j})$ and $e_{2}=\frac{1}{2}(1-\mathbf{j})$. This basis is such that

$$
\mathrm{e}_{1}^{2}=\mathrm{e}_{1}, \quad \mathrm{e}_{2}^{2}=\mathrm{e}_{2} \quad \text { and } \quad \mathrm{e}_{1} \mathrm{e}_{2}=\mathrm{e}_{2} \mathrm{e}_{1}=0 .
$$

\footnotetext{
${ }^{3}$ Also referred to as split complex numbers, hypercomplex numbers, double numbers, real tessarines or Lorentz numbers.
} 
Expressing $\mathrm{q}$ and $\mathrm{c}$ in this basis, we get $\mathrm{q}=x \mathrm{e}_{1}+y \mathrm{e}_{2}$ and $\mathrm{c}=a \mathrm{e}_{1}+b \mathrm{e}_{2}$, where

$$
x=q_{0}+q_{2}, \quad y=q_{0}-q_{2}, \quad a=c_{0}+c_{2} \quad \text { and } \quad b=c_{0}-c_{2} .
$$

Hence,

$$
\mathrm{q}^{2}+\mathrm{c}=\left(x \mathrm{e}_{1}+y \mathrm{e}_{2}\right)^{2}+a \mathrm{e}_{1}+b \mathrm{e}_{2}=\left(x^{2}+a\right) \mathrm{e}_{1}+\left(y^{2}+b\right) \mathrm{e}_{2} .
$$

This shows that the dynamics of the quadratic map $f_{\mathrm{c}}$ in $\mathbb{P}$ reduces to the combination of the dynamics of two real quadratic maps. For example, all the fixed points in $\mathbb{P}$ are of the form

$$
x_{1} \mathrm{e}_{1}+x_{2} \mathrm{e}_{2}=\frac{1}{2}\left(x_{1}+x_{2}\right)+\frac{1}{2}\left(x_{1}-x_{2}\right) \mathbf{j},
$$

where $x_{1}$ is a fixed point of the real quadratic map $f_{a}$ and $x_{2}$ is a fixed point of the real quadratic map $f_{b}$. As for periodic points of period two, it is clear that they are of the form (9) above with $x_{1}$ and $x_{2}$ chosen either as periodic points of period two or fixed points of $f_{a}$ and $f_{b}$, respectively, with the restriction that they cannot be chosen simultaneously as fixed points. Note also that, when $\mathrm{c}=c_{0} \in \mathbb{R}$, the two quadratic maps $f_{a}$ and $f_{b}$ coincide with $f_{c_{0}}$.

\subsubsection{Dynamics in $\mathbb{D}$}

We now analyze the case where both $\mathrm{q}$ and $\mathrm{c}$ are in $\mathbb{D}$, i.e. we consider $\mathrm{q}=q_{0}+a \mathbf{i}+a \mathbf{j}$ and $\mathrm{c}=c_{0}+b \mathbf{i}+b \mathbf{j}$ with $q_{0}, a, c_{0}, b \in \mathbb{R}$. Let us write $f_{\mathrm{c}}^{n}(\mathrm{q}) ; n=0,1,2, \ldots$ as

$$
f_{\mathrm{c}}^{n}(\mathrm{q})=q_{0}^{[n]}+a^{[n]} \mathbf{i}+a^{[n]} \mathbf{j}
$$

with $q_{0}^{[0]}=q_{0}$ and $a^{[0]}=a$. It is easily shown, by induction, that, for $n=1,2, \ldots$, we have

$$
q_{0}^{[n]}=\left(q_{0}^{[n-1]}\right)^{2}+c_{0},
$$

and

$$
a^{[n]}=A^{[n]} a+B^{[n]},
$$

where $A^{[n]}$ and $B^{[n]}$ are given recursively by

$$
\begin{aligned}
& A^{[n]}=2 q_{0}^{[n-1]} A^{[n-1]}, \quad A^{[0]}=1, \\
& B^{[n]}=2 q_{0}^{[n-1]} B^{[n-1]}+b, \quad B^{[0]}=0 \text {. }
\end{aligned}
$$

Note that equation (10) shows that $q_{0}^{[n]}=f_{c_{0}}^{n}\left(q_{0}\right)$, which, in turn, guarantees that $A^{[n]}$ and $B^{[n]}$ are functions of $c$ and $q_{0}$ only (i.e. do not involve $a$ ).

Hence, we see that $\mathrm{q}=q_{0}+a \mathbf{i}+a \mathbf{j}$ satisfies $f_{\mathrm{c}}^{n}(\mathrm{q})=\mathrm{q}$ if and only if $f_{c_{0}}^{n}\left(q_{0}\right)=q_{0}$ and $a$ satisfies the linear equation $\left(A^{[n]}-1\right) a=-B^{[n]}$. By using the above results for the particular choices of $n=1$ and $n=2$ it is simple to conclude that, in this case, we have the following two fixed points, for $c=c_{0}+b \mathbf{i}+b \mathbf{j}$ with $c_{0}<\frac{1}{4}$,

$$
q=\frac{1}{2}\left(1-\sqrt{1-4 c_{0}}\right)+\frac{b}{\sqrt{1-4 c_{0}}}(\mathbf{i}+\mathbf{j})
$$

and

$$
\mathrm{q}=\frac{1}{2}\left(1+\sqrt{1-4 c_{0}}\right)-\frac{b}{\sqrt{1-4 c_{0}}}(\mathbf{i}+\mathbf{j}),
$$

and the following 2-cycle $\{\mathrm{q}, \mathrm{p}\}$, for $\mathrm{c}=c_{0}+b \mathbf{i}+b \mathbf{j}$, with $c_{0}<-\frac{3}{4}$,

$$
\mathrm{q}=\frac{1}{2}\left(-1+\sqrt{-3-4 c_{0}}\right)-\frac{b}{\sqrt{-3-4 c_{0}}}(\mathbf{i}+\mathbf{j}),
$$


and

$$
\mathrm{p}=\frac{1}{2}\left(-1-\sqrt{-3-4 c_{0}}\right)+\frac{b}{\sqrt{-3-4 c_{0}}}(\mathbf{i}+\mathbf{j})
$$

From the study conducted by Fishback [11] we can conclude that the behavior, in terms of stability, of the above points accompanies the behavior of the corresponding real parts: the fixed point (11)(defined for $\left.c_{0}<1 / 4\right)$ is attractive for $c_{0}>-3 / 4$ and repelling for $c_{0}<-3 / 4$ and the fixed point (12) is repelling; the 2-cycle given by the points (13)-(14) (defined for $c_{0}<-3 / 4$ ) is attractive for $c_{0}>-5 / 4$ and repelling for $c_{0}<-5 / 4$. Note also that, when $\mathrm{c}=c_{0}=\frac{1}{4}$, any point of the form

$$
\mathrm{q}=\frac{1}{2}+a \mathbf{i}+a \mathbf{j}, \quad a \in \mathbb{R}
$$

is a fixed point of $f_{\mathrm{c}}$. Similarly, for $\mathrm{c}=c_{0}=-\frac{3}{4}$, any two-points $\{\mathrm{q}, \mathrm{p}\}$ of the form

$$
\mathrm{q}=-\frac{1}{2}+a \mathbf{i}+a \mathbf{j}, \quad \mathrm{p}=-\frac{1}{2}-a \mathbf{i}-a \mathbf{j},
$$

with $a \in \mathbb{R}, a \neq 0$, form a 2 -cycle.

\section{4 “Non-canonical” Fixed Points and Periodic Points of Period Two}

In this section we determine the fixed points and periodic points of period two for the quadratic map $f_{\mathrm{c}}$. Since we are only considering parameters $\mathrm{c}$ with one of the forms $\mathcal{C}_{0}-\mathcal{C}_{3}$, choosing $\mathrm{q} \in \operatorname{span}_{\mathbb{R}}(1, c)$ will lead to dynamics lying in one of the cycle planes $\mathbb{C}, \mathbb{P}$ or $\mathbb{D}$, which, as shown in the previous section, reduces, in a certain way, to the well-studied cases of the dynamics in $\mathbb{C}$ or $\mathbb{R}$. Hence, in what follows, we will leave from our discussion the case where $q \in \operatorname{span}_{\mathbb{R}}(1, c)$, concentrating on "non-canonical" cases where some real novelty may appear.

We observe that, in the special case where $\mathrm{c}=c_{0} \in \mathbb{R}$, we have that a point $\mathrm{q} \in \mathbb{H}_{\text {coq }}$ is a periodic point of period $k$ of $f_{c_{0}}$ if and only if, for any invertible coquaternion $\mathrm{h}$, the coquaternion $\mathrm{h}^{-1} \mathrm{qh}$ is also a periodic point of order $k$ of $f_{c_{0}}$, since $f_{c_{0}}\left(\mathrm{~h}^{-1} \mathrm{qh}\right)=\mathrm{h}^{-1} f_{c_{0}}(\mathrm{q}) \mathrm{h}$. Thus, we have that a coquaternion $\mathrm{q}$ is a (non-real) periodic point of period $k$ of the map $f_{c_{0}}$ if and only if it belongs to the similarity class of $\tilde{\mathrm{q}}$, with $\tilde{\mathrm{q}}$ a periodic point of order $k$ of $f_{c_{0}}$ with the special form $\mathcal{C}_{1}, \mathcal{C}_{2}$ or $\mathcal{C}_{3}$.

\subsection{Fixed points}

Let $\mathrm{q}=q_{0}+q_{1} \mathbf{i}+q_{2} \mathbf{j}+q_{3} \mathbf{k}$ and $\mathrm{c}=c_{0}+c_{1} \mathbf{i}+c_{2} \mathbf{j}+c_{3} \mathbf{k}$ be coquaternions. From (6) we see that $\mathrm{q}$ is a fixed point of $f_{\mathrm{c}}$ if and only if

$$
(\alpha-1) \mathrm{q}+\gamma=-\mathrm{c}
$$

where $\alpha=\operatorname{tr} q=2 q_{0}$ and $\gamma=-\operatorname{det} q$. Noting that $\alpha \neq 1$ leads to $\mathrm{q} \in \operatorname{span}_{\mathbb{R}}(1, \mathrm{c})$, we will only consider the case $\alpha=1$, i.e. simply analyze the equation $\gamma=-$ c. Since $\gamma$ is a real number, we conclude that, if $c \in \mathbb{H}_{\text {coq }} \backslash \mathbb{R}$, there are no fixed points. On the other hand, for $\mathrm{c}=c_{0} \in \mathbb{R}$, as observed previously, to determine the sets of non-real fixed points, we will only have to determine the fixed points with the special forms $\mathcal{C}_{1}, \mathcal{C}_{2}$ or $\mathcal{C}_{3}$ and construct the corresponding similarity classes.

The only fixed point of the form $\mathcal{C}_{1}$ occurs when $c_{0}>\frac{1}{4}$ and is given by $x=\frac{1}{2}+\frac{1}{2} \sqrt{4 c_{0}-1} \mathbf{i}$; cf. (7). Also, it is simple to see that the only fixed point of $f_{c_{0}}$ of the form $\mathcal{C}_{2}$ occurs for $c_{0}<\frac{1}{4}$ and is given by $x=\frac{1}{2}+\frac{1}{2} \sqrt{1-4 c_{0}} \mathbf{j}$ (this point is obtained by using formula (9) with the choice $x_{1}=\frac{1}{2}\left(1+\sqrt{1-4 c_{0}}\right)$ and $\left.x_{2}=\frac{1}{2}\left(1-\sqrt{1-4 c_{0}}\right)\right)$. Finally, $x=\frac{1}{2}+\mathbf{i}+\mathbf{j}$ is the only fixed point of the form $\mathcal{C}_{3}$ and occurs when $c_{0}=\frac{1}{4}$; see (15). Hence, in this case, we have the following three types of sets of (non-real) fixed points, depending 


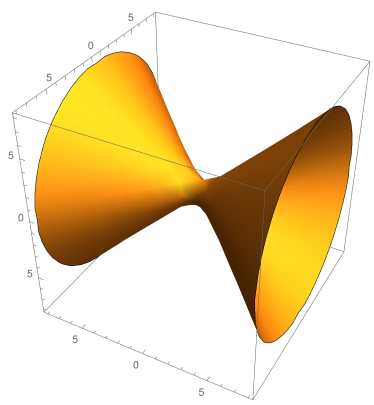

$(a)$

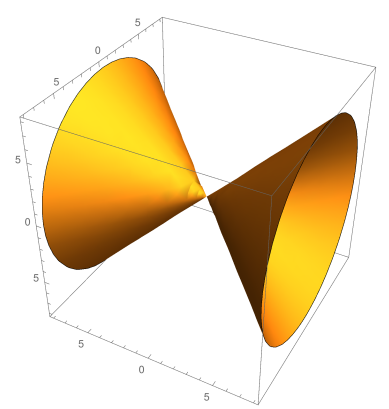

(b)

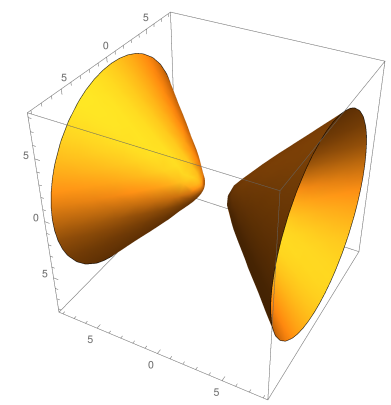

(c)

Figure 1: Plots, in the hyperplane $q_{0}=1 / 2$, of the fixed points corresponding to the case $q_{0}=1 / 2$ and $\mathrm{c}=c_{0} \in \mathbb{R} .(a) c_{0}<\frac{1}{4} ;(b) c_{0}=\frac{1}{4} ;(c) c_{0}>\frac{1}{4}$.

on the value of $c_{0}$ :

$$
\begin{array}{ll}
\mathcal{F}_{1}=\left[\frac{1}{2}+\frac{1}{2} \sqrt{1-4 c_{0}} \mathbf{j}\right], & \text { if } \quad c_{0}<\frac{1}{4}, \\
\mathcal{F}_{2}=\left[\frac{1}{2}+\mathbf{i}+\mathbf{j}\right], & \text { if } \quad c_{0}=\frac{1}{4}, \\
\mathcal{F}_{3}=\left[\frac{1}{2}+\frac{1}{2} \sqrt{4 c_{0}-1} \mathbf{i}\right], & \text { if } \quad c_{0}>\frac{1}{4} .
\end{array}
$$

Note that, as observed before, any of the above sets can be identified with an hyperboloid in the hyperplane $\left\{\left(q_{0}, q_{1}, q_{2}, q_{3}\right) \in \mathbb{R}^{4}: q_{0}=\frac{1}{2}\right\}: \mathcal{F}_{1}$ is an hyperboloid of one-sheet, $\mathcal{F}_{2}$ is a degenerate hyperboloid (cone) and $\mathcal{F}_{3}$ is an hyperboloid of two-sheets see: Fig. 1.

The plots in Fig. 1 were obtained by fixing $q_{0}=\frac{1}{2}$ and hence cannot display some of the known fixed points in the complex plane; in order to have a picture in which these points are easily shown, we can fix $q_{3}=0$, instead. In Fig. 2 we present plots obtained in this manner, for several values of the parameter $c_{0}$. The known fixed points of the dynamics in $\mathbb{C}$ are identified with black points and the fixed points not in $\mathbb{C}$ are given by blue lines (hyperbolas resulting from the intersection of the hyperboloids $\mathcal{F}_{i}$ with the hyperplane $\left.H_{3}=\left\{\left(q_{0}, q_{1}, q_{2}, q_{3}\right) \in \mathbb{R}^{4}: q_{3}=0\right\}\right)$; the real and imaginary axis are identified with gray lines.

\subsection{Periodic points of period two}

We want to study now the conditions under which $q \in \mathbb{H}_{\text {coq }}$ is a periodic point of period two of $f_{\mathrm{c}}$. By using Lemma 3.1, we have $\mathrm{p}=f_{\mathrm{c}}(\mathrm{q})=(\operatorname{tr} \mathrm{q}) \mathrm{q}-\operatorname{det} \mathrm{q}+\mathrm{c}$ and

$$
f_{\mathrm{c}}^{2}(\mathrm{q})=f_{\mathrm{c}}(\mathrm{p})=\alpha^{\prime} \mathrm{q}+\beta^{\prime} \mathrm{c}+\gamma^{\prime}
$$

with

$$
\alpha^{\prime}=\operatorname{tr} \mathrm{p} \operatorname{tr} \mathrm{q}, \quad \beta^{\prime}=1+\operatorname{tr} \mathrm{p}, \quad \gamma^{\prime}=-\operatorname{tr} \mathrm{p} \operatorname{det} \mathrm{q}-\operatorname{det} \mathrm{p} .
$$

Hence, we have that $\{\mathrm{q}, \mathrm{p}\}$ is a 2-cycle if and only if $\left(\alpha^{\prime}-1\right) \mathrm{q}+\gamma^{\prime}=-\beta^{\prime} \mathrm{c}$, with $\alpha^{\prime}, \beta^{\prime}, \gamma^{\prime}$ given by (20) and $\mathrm{p} \neq \mathrm{q}$. In analogy with the study conducted for the fixed points, since $\alpha^{\prime} \neq 1$ implies that $\mathrm{q} \in \operatorname{span}_{\mathbb{R}}(1, \mathrm{c})$, we will assume now that $\alpha^{\prime}=1$, in which case we obtain the equation

$$
\gamma^{\prime}=-\beta^{\prime} \mathrm{c}
$$

We will now analyze separately two different situations: $c \in \mathbb{R}$ and $\mathrm{c} \in \mathbb{H}_{\text {coq }} \backslash \mathbb{R}$. 


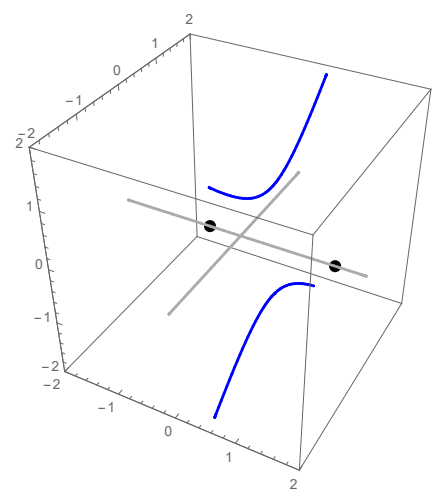

(a)

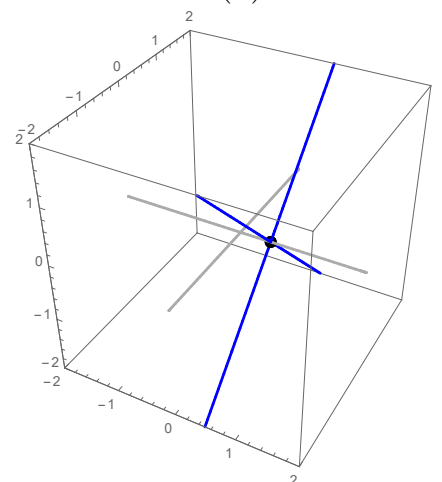

(d)

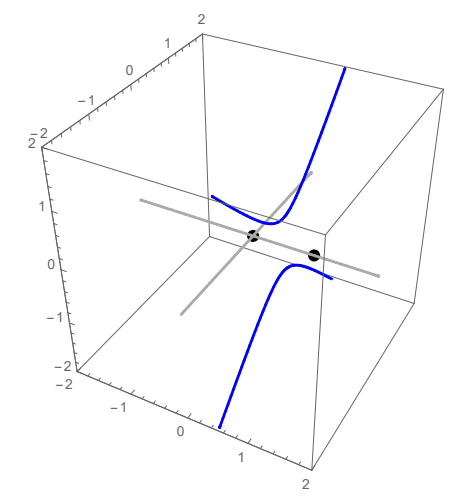

(b)

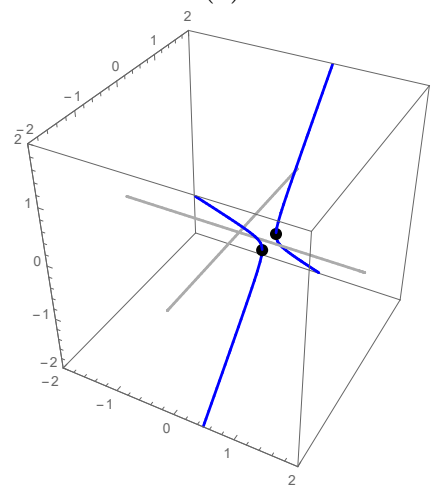

(e)

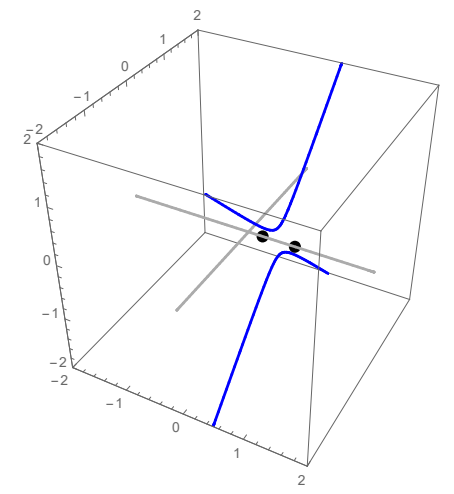

(c)

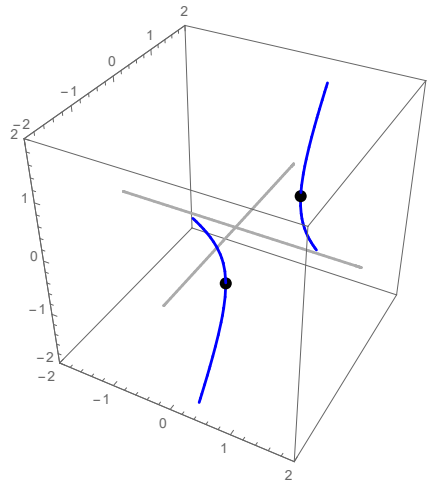

$(f)$

Figure 2: Plots, in the hyperplane $q_{3}=0$, of the sets of fixed points corresponding to the case $\mathrm{c}=c_{0} \in \mathbb{R}$; the real and complex fixed points are identified with black points and the fixed points not in $\mathbb{C}$ form the blue lines; the gray lines represent the real and imaginary axes. $(a) c_{0}=-0.8 ;(b) c_{0}=0.0 ;(c) c_{0}=0.18 ;(d)$ $c_{0}=0.25 ;(e) \quad c_{0}=0.3$ and $(f) c_{0}=1.7$.

\subsubsection{Case $c \in \mathbb{R}$}

As referred previously, to determine the (non-real) periodic points of period two of $f_{\mathrm{c}}$ with $\mathrm{c}=c_{0} \in \mathbb{R}$, we only have to identify the periodic points of period two of this map with any of the special forms $\mathcal{C}_{1}-\mathcal{C}_{3}$. By using a procedure similar to the one described for the case of fixed points, it can be shown easily that we have the following seven sets of periodic-points of period two:

$$
\mathcal{P}_{i}=\left[q_{0}^{(i)}+q_{2}^{(i)} \mathbf{j}\right] ; i=1,2,3,4,5, \quad c_{0}<-\frac{3}{4},
$$

where

$$
\begin{cases}q_{0}^{(1)}=-\frac{1}{2}, & q_{2}^{(1)}=\frac{1}{2} \sqrt{-3-4 c_{0}} \\ q_{0}^{(2)}=\frac{1}{4}\left(-\sqrt{-3-4 c_{0}}-\sqrt{1-4 c_{0}}\right), & q_{2}^{(2)}=\frac{1}{4}\left(2+\sqrt{-3-4 c_{0}}-\sqrt{1-4 c_{0}}\right), \\ q_{0}^{(3)}=\frac{1}{4}\left(\sqrt{-3-4 c_{0}}-\sqrt{1-4 c_{0}}\right), & q_{2}^{(3)}=-\frac{1}{4}\left(2-\sqrt{-3-4 c_{0}}-\sqrt{1-4 c_{0}}\right), \\ q_{0}^{(4)}=\frac{1}{4}\left(-\sqrt{-3-4 c_{0}}+\sqrt{1-4 c_{0}}\right), & q_{2}^{(4)}=\frac{1}{4}\left(2+\sqrt{-3-4 c_{0}}+\sqrt{1-4 c_{0}}\right), \\ q_{0}^{(5)}=\frac{1}{4}\left(\sqrt{-3-4 c_{0}}+\sqrt{1-4 c_{0}}\right), & q_{2}^{(5)}=\frac{1}{4}\left(2-\sqrt{-3-4 c_{0}}+\sqrt{1-4 c_{0}}\right), \\ \mathcal{P}_{6}=\left[-\frac{1}{2}+\mathbf{i}+\mathbf{j}\right], & c_{0}=-\frac{3}{4},\end{cases}
$$




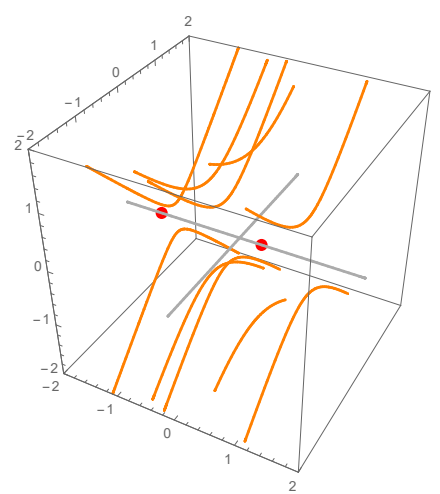

(a)

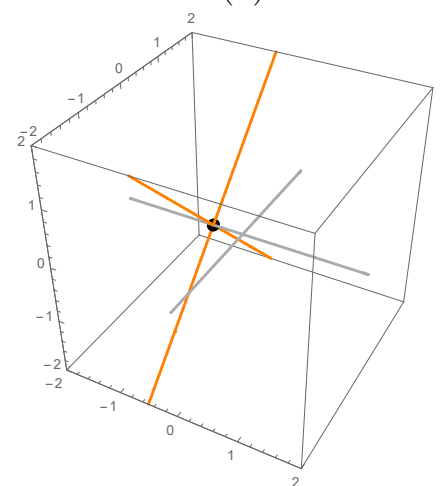

(d)

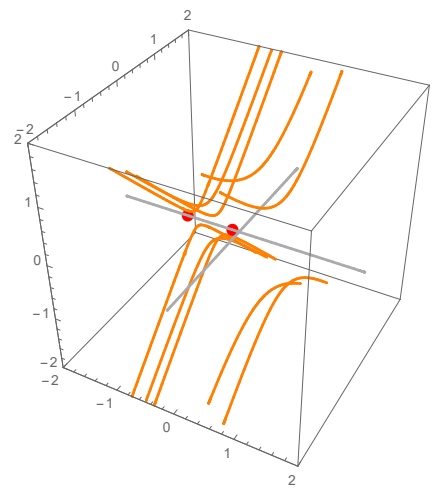

(b)

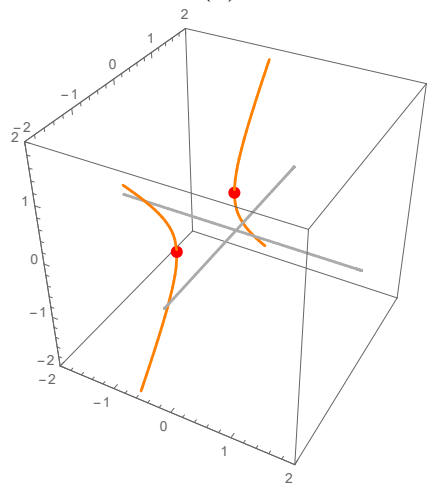

(e)

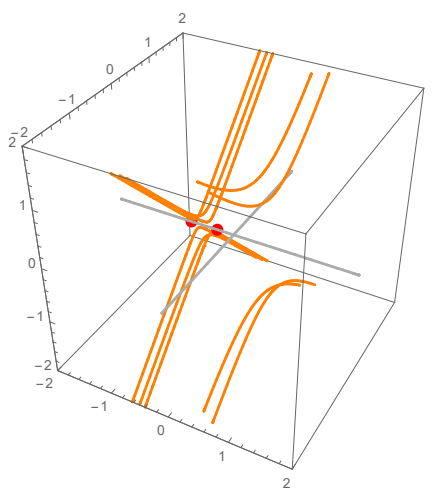

(c)

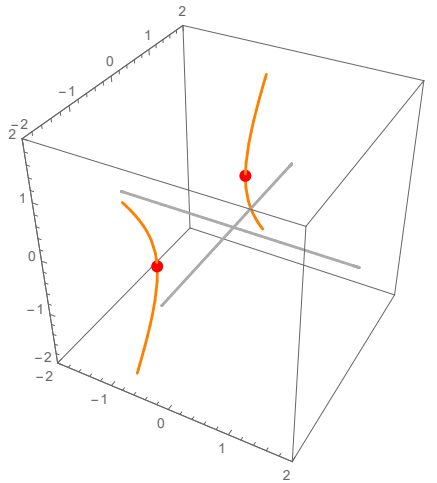

$(f)$

Figure 3: Plots, in the hyperplane $q_{3}=0$, of the sets of periodic points of period two corresponding to parameters $\mathrm{c}=c_{0} \in \mathbb{R}$, for different values of $c_{0}$. The known periodic points in the complex plane are identified with red points and the periodic points not in $\mathbb{C}$ are given by orange lines; the black point in subplot $(d)$ is a fixed point; the real and imaginary axes are identified with gray lines; $(a) c_{0}=-1.5 ;(b) c_{0}=-0.9$; (c) $c_{0}=-0.8 ;(d) c_{0}=-0.75 ;(e) c_{0}=0.0 ;(f) c_{0}=1.0$.

and

$$
\mathcal{P}_{7}=\left[-\frac{1}{2}+\frac{1}{2} \sqrt{3+4 c_{0}} \mathbf{i}\right], \quad c_{0}>-\frac{3}{4} .
$$

Note that, for $\mathrm{q} \in \mathcal{P}_{i} ; i=1,6,7$, we have $\mathrm{p}=f(\mathrm{q})=\overline{\mathrm{q}} \in \mathcal{P}_{i}$ and, since there are no real points in any of these sets, the condition $f(\mathrm{q}) \neq \mathrm{q}$ is verified. For $\mathrm{q} \in \mathcal{P}_{i} ; i=2,3,4,5$, we have $\mathrm{p}=p_{0}+p_{1} \mathbf{i}+p_{2} \mathbf{j}+p_{3} \mathbf{k}$ with

$$
p_{0}=\frac{1}{4 q_{0}}, \quad p_{1}=2 q_{0} q_{1}, \quad p_{2}=2 q_{0} q_{2}, \quad p_{3}=2 q_{0} q_{3} .
$$

It can easily be verified that, for $\mathrm{q} \in \mathcal{P}_{2}, \mathrm{p} \in \mathcal{P}_{3}$ and, for $\mathrm{q} \in \mathcal{P}_{4}, \mathrm{p} \in \mathcal{P}_{5}$. In analogy to what we did in the case of fixed points, in Fig. 3 we present plots, obtained by fixing $q_{3}=0$, of the periodic points of period two corresponding to parameters $\mathrm{c}=c_{0} \in \mathbb{R}$, for different values of $c_{0}$. The known periodic points of period two in the complex plane are identified with red points and the periodic points not in $\mathbb{C}$ are given by orange lines (resulting from the intersection of the sets $\mathcal{P}_{i} ; i=1, \ldots, 7$, with the hyperplane $q_{3}=0$ ); the real and imaginary axes are identified with gray lines.

\subsubsection{Case $c \in \mathbb{H}_{\text {coq }} \backslash \mathbb{R}$}

As $\gamma^{\prime}$ and $\beta^{\prime}$ are real quantities, if $c \in \mathbb{H}_{\text {coq }} \backslash \mathbb{R}$, Eq. (21) is satisfied if and only if $\beta^{\prime}=\gamma^{\prime}=0$. It follows immediately from Eq. (1) that

$$
\operatorname{tr} \mathrm{p}=2\left(q_{0}^{2}-q_{1}^{2}+q_{2}^{2}+q_{3}^{2}+c_{0}\right)
$$


and, from Eq. (2),

$$
\operatorname{det} \mathrm{p}=\left(q_{0}^{2}-q_{1}^{2}+q_{2}^{2}+q_{3}^{2}+c_{0}\right)^{2}+\left(2 q_{0} q_{1}+c_{1}\right)^{2}-\left(2 q_{0} q_{2}+c_{2}\right)^{2}-\left(2 q_{0} q_{3}+c_{3}\right)^{2} .
$$

Now, from the expressions of $\beta^{\prime}$ and $\alpha^{\prime}$, we have

$$
\begin{aligned}
\beta^{\prime}=0 \text { and } \alpha^{\prime}=1 & \Longleftrightarrow \operatorname{tr} \mathrm{q}=\operatorname{tr} \mathrm{p}=-1 \\
& \Longleftrightarrow\left\{\begin{array}{l}
q_{0}=-\frac{1}{2} \\
q_{1}^{2}-q_{2}^{2}-q_{3}^{2}=\frac{3+4 c_{0}}{4}
\end{array}\right.
\end{aligned}
$$

For $q_{0}=-\frac{1}{2}$ and $\operatorname{tr} \mathrm{p}=-1$, the expression of the determinant of $\mathrm{p}$ given by (26) simplifies to

$$
\operatorname{det} \mathrm{p}=\frac{1}{4}+\left(c_{1}-q_{1}\right)^{2}-\left(c_{2}-q_{2}\right)^{2}-\left(c_{3}-q_{3}\right)^{2} .
$$

Hence, in this case, we have

$$
\begin{aligned}
\gamma^{\prime}=0 & \Longleftrightarrow \operatorname{det} \mathrm{q}-\operatorname{det} \mathrm{p}=0 \\
& \Longleftrightarrow\left(2 q_{1}-c_{1}\right) c_{1}-\left(2 q_{2}-c_{2}\right) c_{2}-\left(2 q_{3}-c_{3}\right) c_{3}=0 .
\end{aligned}
$$

(i) Case $\mathrm{c}$ with the form $\mathcal{C}_{1}$ If $\mathrm{c}=c_{0}+c_{1} \mathbf{i}$, with $c_{1}>0$, Eq. (28) is satisfied if and only if $q_{1}=c_{1} / 2$. But then, the second of equations (27) reduces to $q_{2}^{2}+q_{3}^{2}=\frac{c_{1}^{2}-4 c_{0}-3}{4}$. In this case, we thus have the following circle of periodic points of period two (in the plane $q_{0}=-\frac{1}{2}$ and $q_{1}=\frac{c_{1}}{2}$ ):

$$
\mathcal{P}_{8}=\left\{-\frac{1}{2}+\frac{c_{1}}{2} \mathbf{i}+q_{2} \mathbf{j}+q_{3} \mathbf{k}: q_{2}^{2}+q_{3}^{2}=\frac{c_{1}^{2}-4 c_{0}-3}{4}\right\}, \quad c_{1}^{2}>4 c_{0}+3 .
$$

For $\mathrm{q} \in \mathcal{P}_{8}$, we have $f_{\mathrm{c}}(\mathrm{q})=-\frac{1}{2}+\frac{c_{1}}{2} \mathbf{i}-q_{2} \mathbf{j}-q_{3} \mathbf{k} \neq \mathrm{q}$.

(ii) Case c with the form $\mathcal{C}_{2} \quad$ A study analogous to the one conducted for the previous case shows that, when $\mathrm{c}=c_{0}+c_{2} \mathbf{j}, c_{2}>0$, we have the following set of periodic points of period two:

$$
\mathcal{P}_{9}=\left\{-\frac{1}{2}+q_{1} \mathbf{i}+\frac{c_{2}}{2} \mathbf{j}+q_{3} \mathbf{k}: q_{1}^{2}-q_{3}^{2}=\frac{c_{2}^{2}+4 c_{0}+3}{4}\right\} \backslash\left\{-\frac{1}{2}+\frac{c_{2}}{2} \mathbf{j}\right\} .
$$

This is an hyperbola, in the plane $q_{0}=-\frac{1}{2}$ and $q_{2}=\frac{c_{2}}{2}$, which, when $c_{2}^{2}+4 c_{0}+3=0$, degenerates into pair lines (from which we exclude the intersection point $\mathrm{q}=-\frac{1}{2}+\frac{c_{2}}{2} \mathbf{j}$, which is a fixed point for $f_{\mathrm{c}}$, in this case). For $\mathrm{q}=-\frac{1}{2}+q_{1} \mathbf{i}+\frac{c_{2}}{2} \mathbf{j}+q_{3} \mathbf{k}$ in the above set, we have $f_{\mathrm{c}}(\mathrm{q})=-\frac{1}{2}-q_{1} \mathbf{i}+\frac{c_{2}}{2} \mathbf{j}-q_{3} \mathbf{k}$.

(iii) Case $\mathrm{c}$ with the form $\mathcal{C}_{3}$ When $\mathrm{c}=c_{0}+\mathbf{i}+\mathbf{j}$, following a similar procedure to the two previous cases, we conclude that we have the following set of periodic points of period two:

$$
\mathcal{P}_{10}=\left\{-\frac{1}{2}+q_{1} \mathbf{i}+q_{1} \mathbf{j} \pm \frac{1}{2} \sqrt{-4 c_{0}-3} \mathbf{k}\right\} \backslash\left\{-\frac{1}{2}+\frac{1}{2} \mathbf{i}+\frac{1}{2} \mathbf{j}\right\}, \quad c_{0} \leq-\frac{3}{4} .
$$

This is a pair of parallel lines which, when $c_{0}=-\frac{3}{4}$, become coincident; the image of a point $\mathrm{q}=-\frac{1}{2}+q_{1} \mathbf{i}+$ $q_{1} \mathbf{j}+\frac{1}{2} \sqrt{-4 c_{0}-3} \mathbf{k}$ in one of the lines is the point $-\frac{1}{2}+\left(1-q_{1}\right) \mathbf{i}+\left(1-q_{1}\right) \mathbf{j}-\frac{1}{2} \sqrt{-4 c_{0}-3} \mathbf{k}$ in the other line; when $c_{0}=-\frac{3}{4}$ and $q_{1}=\frac{1}{2}$, we have a fixed point of the map $f_{\mathrm{c}}$.

\section{Stability}

We now want to study the stability of the fixed points and periodic points of period two determined in the previous section. As we have seen, we now have a situation which does not occur in the classical case of the complex quadratic map: the existence of sets of non-isolated periodic points. It is simple to recognize 
that points in these type of sets will never be attractive in the usual sense. Consider, for example, the case of a set of non-isolated fixed points: since, given any point in the set, all its neighborhoods always contain another fixed point, it will be impossible to find a neighborhood of a given point totally formed by points whose dynamics evolve to it. Hence, it becomes necessary to introduce a new notion of attractivity; see e.g. [3]. We propose to adopt the following definition.

Definition 5.1. A set $\mathcal{S}$ of non-isolated periodic points of period $n \in \mathbb{N}$ of the coquaternionic map $f_{\mathrm{c}}$ is said to be attractive if, given any coquaternion q sufficiently close to the set $\mathcal{S}$, the sequence of iterates $\left(\left(f_{\mathrm{c}}^{n}\right)^{k}(\mathrm{q})\right)_{k}$ converges to a point belonging to $\mathcal{S}$.

Remark 5.1. In the above definition it is understood that the distance from a coquaternion $\mathrm{q}$ to the set $\mathcal{S}$ is measured by $d(\mathrm{q}, \mathcal{S})=\inf _{\mathrm{s} \in \mathcal{S}}\|\mathrm{q}-\mathrm{s}\|$ with $\|$.$\| any norm in the space \mathbb{R}^{4}$, for example the Euclidean norm; the convergence of the sequence $\left(\left(f_{\mathrm{c}}^{n}\right)^{k}(\mathrm{q})\right)_{k}$ is to be understood as convergence with respect to the same norm.

Let $\mathcal{S}=\left[\mathrm{q}_{\mathcal{S}}\right]$ be any of the sets of fixed points given by (17)-(19) or any of the sets of periodic points of period two given by (22)-(25)(all of them corresponding to a real parameter $\mathrm{c}=c_{0}$ ) with the representative $q_{\mathcal{S}}$ chosen in canonical form. It is a trivial matter to conclude that if $\mathrm{q}_{\mathcal{S}}$ is not an attractive point for the map $f_{c_{0}}$ restricted to the cycle plane where qs lies, then the corresponding set $\mathcal{S}$ cannot be an attractive set. An analysis of the stability of each of the representatives of the sets mentioned above shows that the only one which is attractive is the coquaternion $\mathrm{q}_{\mathcal{P}_{1}}=-\frac{1}{2}+\frac{1}{2} \sqrt{-3-4 c_{0}} \mathbf{j}$, for values of $c_{0}$ such that $-5 / 4<c_{0}<-3 / 4$. Thus, we immediately conclude that none of the sets of fixed points $\mathcal{F}_{1}-\mathcal{F}_{3}$ nor any of the sets of periodic points of period two $\mathcal{P}_{2}-\mathcal{P}_{7}$ is attractive. We will now discuss the attractivity of the other sets.

Since there is no appropriate notion of derivative for coquaternionic maps, the natural approach to study stability of a given periodic point of period $n$ is to consider the function $f_{c}^{n}$ as a function from $\mathbb{R}^{4}$ into $\mathbb{R}^{4}$ and discuss the magnitude of the modulus of eigenvalues of the respective Jacobian matrix. As it is well known, if all the eigenvalues of this matrix have modulus less than one, then the periodic point is attractive and, if there exists one eigenvalue with modulus greater than one, the point is not attractive. In the case of sets of non-isolated periodic points we are studying here, the situation where all the eigenvalues of the Jacobian matrix have absolute value less than one will never occur, since, as observed before, it is impossible to have attractivity in the usual sense. However, it is expected that some relationship exists between the magnitude of the eigenvalues of the Jacobian matrix and the attractivity/non-attractivity, in the new sense introduced above, of the sets considered. The results that we now present led us to conclude that this is, in fact true.

In what follows, we will denote by $\mathbf{J}_{f_{\mathrm{c}}^{2}}(\mathrm{q})$ the Jacobian matrix of the map $f_{\mathrm{c}}^{2}$ computed at a given point $\mathrm{q}$; when the parameter $\mathrm{c}$ is implicit from the context, we will also use the abbreviate notation $\mathbf{J}_{2}(\mathrm{q})$.

\subsection{Stability of the set $\mathcal{P}_{1}$}

Consider first the analysis of the stability of the set

$$
\mathcal{P}_{1}=\left[-\frac{1}{2}+\frac{1}{2} \sqrt{-3-4 c_{0}} \mathbf{j}\right] .
$$

Note that this is a set of periodic points of period two for the quadratic map associated with a real parameter $c_{0}$ satisfying $c_{0}<-3 / 4$. For $c_{0} \leq-5 / 4$, the representative of the set is not an attractive point of the map $f_{c_{0}}$ restricted to the plane $\mathbb{P}$, and hence the set is not attractive. We now consider the case $-5 / 4<c_{0}<-3 / 4$. From the expression of the set $\mathcal{P}_{1}$, it is clear that coquaternions belonging to this set are characterized by having a real part equal to $-1 / 2$ and a vector part whose determinant is equal to $c_{0}+\frac{3}{4}<0$. Let $q$ be a point close enough to the set $\mathcal{P}_{1}$ so that we still have $\operatorname{det}(\operatorname{vec} \mathrm{q})<0$ and let $\mathrm{q}^{*}$ be the point in $\mathcal{P}_{1}$ given by

$$
\mathrm{q}^{*}=-\frac{1}{2}+\alpha \operatorname{vec} \mathrm{q}, \quad \text { with } \alpha=\sqrt{\frac{c_{0}+\frac{3}{4}}{\operatorname{det}(\operatorname{vec} \mathrm{q})}} .
$$

It can be shown that the eigenvalues of the Jacobian matrix of $f_{c_{0}}^{2}$ at the point $\mathrm{q}^{*}$ are given by

$$
\lambda_{1}=\lambda_{2}=1, \quad \lambda_{3}=\lambda_{4}=4\left(c_{0}+1\right),
$$


with associated eigenvectors $\mathrm{v}_{1}^{*}=\left(0, q_{3}^{*}, 0, q_{1}^{*}\right), \mathrm{v}_{2}^{*}=\left(0, q_{2}^{*}, q_{1}^{*}, 0\right), \mathrm{v}_{3}^{*}=\left(0, q_{1}^{*}, q_{2}^{*}, q_{3}^{*}\right)$ and $\mathrm{v}_{4}^{*}=(1,0,0,0)$. Since vec $\mathrm{q}^{*}=\alpha$ vec $\mathrm{q}$, it is clear that we can also choose as eigenvectors the vectors given by

$$
\mathrm{v}_{1}=\left(0, q_{3}, 0, q_{1}\right), \quad \mathrm{v}_{2}=\left(0, q_{2}, q_{1}, 0\right), \quad \mathrm{v}_{3}=\left(0, q_{1}, q_{2}, q_{3}\right), \quad \mathrm{v}_{4}=(1,0,0,0) .
$$

Remark 5.2. We assumed here, for simplicity, that the point q considered is such that $q_{i} \neq 0 ; i=1,2,3 ;$ for other choices of q, the arguments below have to be slightly modified, but the conclusions still hold.

Note that the vectors $\mathrm{v}_{1}, \mathrm{v}_{2}, \mathrm{v}_{3}, \mathrm{v}_{4}$ are linearly independent, and hence constitute a basis for $\mathbb{R}^{4}$. Because we are considering $\mathrm{c} \in \mathbb{R}$, we have $\mathcal{O}(\mathrm{q}) \subseteq \operatorname{span}_{\mathbb{R}}(1, \mathrm{q})=\operatorname{span}_{\mathbb{R}}(1$, vec $\mathrm{q})$. From the expressions of the eigenvectors given by (30), it is immediate to conclude that $\operatorname{span}_{\mathbb{R}}(1, \operatorname{vec} q)=\operatorname{span}_{\mathbb{R}}\left(\mathrm{v}_{3}, \mathrm{v}_{4}\right)$. This shows that the dynamics of $\mathrm{q}$ is restricted to the plane defined by the two eigenvectors associated with the eigenvalues $\lambda_{3}$ and $\lambda_{4}$; since, for $-5 / 4<c_{0}<-3 / 4$, we have $\left|\lambda_{3}\right|=\left|\lambda_{4}\right|<1$, it becomes clear that $q$ will approach $\mathrm{q}^{*} \in \mathcal{P}_{1}{ }^{4}$ and so we conclude that the set $\mathcal{P}_{1}$ is attractive for these values of $c_{0}$.

Remark 5.3. It is worth observing that the eigenvectors $\mathrm{v}_{1}, \mathrm{v}_{2}$ associated with the eigenvalues $\lambda_{1}=\lambda_{2}=1$ give the directions defining the tangent plane to the set $\mathcal{P}_{1}$ at the point $\mathrm{q}^{*}$.

\subsection{Stability of the set $\mathcal{P}_{8}$}

We now consider the set

$$
\mathcal{P}_{8}=\left\{-\frac{1}{2}+\frac{c_{1}}{2} \mathbf{i}+q_{2} \mathbf{j}+q_{3} \mathbf{k}: q_{2}^{2}+q_{3}^{2}=\frac{c_{1}^{2}-4 c_{0}-3}{4}\right\},
$$

corresponding to the choice of a complex parameter $\mathrm{c}=c_{0}+c_{1} \mathbf{i}$, with $c_{1}>0$ and $c_{1}^{2}>4 c_{0}+3$. The analysis of the stability of this set follows a similar pattern to the analysis of the set $\mathcal{P}_{1}$. It is now convenient to introduce the following notation: for a given coquaternion $q=q_{0}+q_{1} \mathbf{i}+q_{2} \mathbf{j}+q_{3} \mathbf{k}$, we use $\operatorname{vec}_{2} q$ to denote the coquaternion given by $\operatorname{vec}_{2} \mathrm{q}=q_{2} \mathbf{j}+q_{3} \mathbf{k}$.

Let $q$ be a point chosen sufficiently close to the set $\mathcal{P}_{8}$ so that $\operatorname{det}\left(\operatorname{vec}_{2} q\right)<0$ and let $q^{*}$ be the point in $\mathcal{P}_{8}$ given by

$$
\mathrm{q}^{*}=-\frac{1}{2}+\frac{c_{1}}{2} \mathbf{i}+\alpha \operatorname{vec}_{2} \mathrm{q}, \quad \text { with } \alpha=\sqrt{-\frac{\frac{c_{1}^{2}-4 c_{0}-3}{4}}{\operatorname{det}\left(\operatorname{vec}_{2} \mathrm{q}\right)}} .
$$

It can be shown that one of the eigenvalues, $\lambda_{1}$, of $\mathbf{J}_{2}\left(\mathrm{q}^{*}\right)$ is equal to one and has an associated eigenvector which can be chosen as $\mathrm{v}_{1}=\left(0,0,-q_{3}, q_{2}\right)$. The other three eigenvalues $\lambda_{j} ; j=2,3,4$ of $\mathbf{J}_{2}\left(\mathrm{q}^{*}\right)$ are the roots of the cubic polynomial equation

$$
f(\lambda):=\lambda^{3}+A \lambda^{2}+B \lambda+C=0
$$

where

$$
A=4 c_{1}^{2}-8 c_{0}-9, \quad B=\left(2 c_{1}^{2}-4 c_{0}-4\right)^{2}+8\left(c_{0}+1\right), \quad C=-16\left(c_{0}+1\right)^{2},
$$

and have associated eigenvectors which can be chosen as

$$
\mathrm{v}_{i}=\left(A_{i}, B_{i}, q_{2}, q_{3}\right) ; i=2,3,4,
$$

where

$$
A_{i}=-\frac{1}{4 c_{1}^{2} \alpha}\left(-1+\lambda_{i}\right)\left(-4-4 c_{0}+2 c_{1}^{2}+\lambda_{i}\right)
$$

and

$$
B_{i}=\frac{-4-4 c_{0}+c_{1}^{2}+\lambda_{i}}{2 c_{1} \alpha}
$$

We are going to prove that:

(i) one of the eigenvalues $\lambda_{2}, \lambda_{3}$ or $\lambda_{4}$ always has modulus less than one;

\footnotetext{
${ }^{4}$ We have to assume that $\mathrm{q}$ was chosen sufficiently close to the set $\mathcal{P}_{1}$ so that $\mathrm{q}$ is in a sufficiently small neighborhood of $\mathrm{q}^{*}$ where it makes sense to discuss the stability of $\mathrm{q}^{*}$ in terms of the magnitude of the eigenvalues of the Jacobian matrix.
} 
(ii) the two other eigenvalues have modulus less than one for $\left(c_{0}, c_{1}\right)$ in the interior of the region limited by the ellipse

$$
\mathcal{E}:=\left\{\left(c_{0}, c_{1}\right) \in \mathbb{R}^{2}: 16\left(c_{0}+1\right)^{2}+2 c_{1}^{2}=1\right\}
$$

and modulus greater than one outside that ellipse.

First, observe that $f(\lambda)$ can be written as

$$
f(\lambda)=\lambda\left(\lambda+2 c_{1}^{2}-4 c_{0}-4\right)^{2}-\left(\lambda-4 c_{0}-4\right)^{2} .
$$

Taking into account that we are considering $c_{1}>0$ and $c_{1}^{2}>4 c_{0}+3$, it follows easily that

$$
f(1)=4 c_{1}^{2}\left(c_{1}^{2}-4 c_{0}-3\right)>0 .
$$

Since $f(0) \leq 0$ we immediately conclude that there exists a root of $(31)$, say $\lambda_{2}$, satisfying $\lambda_{2} \in[0,1)$, which proves (i).

We now discuss the modulus of the two other eigenvalues $\lambda_{3}$ and $\lambda_{4}$. Since $c_{1}>0$, it follows at once from (34) that $f(\lambda)<0$, for all $\lambda<0$, i.e. real roots of $f(\lambda)$ are non-negative.

We introduce the auxiliary polynomial

$$
g(x):=-x^{3}+B x^{2}-A C x+C^{2}
$$

and remark that the discriminant $\Delta_{g}$ of the polynomial $g$ verifies $\Delta_{g}=C^{2} \Delta_{f}$, where the discriminant of the polynomial $f$ is given by

$$
\Delta_{f}=16 c_{1}^{4}\left(36+64 c_{0}^{3}-136 c_{1}^{2}+49 c_{1}^{4}-8 c_{1}^{6}-32 c_{0}^{2}\left(-5+3 c_{1}^{2}\right)+4 c_{0}\left(33-58 c_{1}^{2}+12 c_{1}^{4}\right)\right) .
$$

We will distinguish three cases, depending on the sign of $\Delta_{f}$.

Case $1-\Delta_{f}<0$ The eigenvalues $\lambda_{3}$ and $\lambda_{4}$ are such that $\lambda_{3}=\overline{\lambda_{4}}=\alpha+i \beta, \beta \neq 0$. Assuming that $C \neq 0$, i.e. $c_{0} \neq-1$, it can easily be shown that $\gamma:=\alpha^{2}+\beta^{2}$ is the (unique) real root of the polynomial equation $g(x)=0$. Moreover, since $g(1)=\left(16\left(c_{0}+1\right)^{2}+2 c_{1}^{2}-1\right)\left(16\left(c_{0}+\frac{3}{4}\right)^{2}+2 c_{1}^{2}\right)$, we conclude that

$$
g(1)<0 \Longleftrightarrow 16\left(c_{0}+1\right)^{2}+2 c_{1}^{2}<1
$$

i.e. $g(1)<0$ if and only if $\left(c_{0}, c_{1}\right)$ is in the interior of the ellipse $\mathcal{E}$ defined in (33), which we will denote by int $\mathcal{E}$. This together with $g(0)=C^{2}>0$ proves that $\gamma=\left|\lambda_{3}\right|^{2}=\left|\lambda_{4}\right|^{2} \in(0,1)$ if and only if $\left(c_{0}, c_{1}\right) \in \operatorname{int} \mathcal{E}$.

If $c_{0}=-1$, then $\Delta_{f}<0$, when $c_{1}>\frac{\sqrt{2}}{4}$, and the eigenvalues $\lambda_{3}, \lambda_{4}$ will have modulus less than one if and only if $c_{1}<\frac{\sqrt{2}}{2}$ (giving points $\left(c_{0}, c_{1}\right) \in \operatorname{int} \mathcal{E}$ ).

Case $2-\Delta_{f}=0$ When $\Delta_{f}$ changes from $\Delta_{f}>0$ to $\Delta_{f}=0$, two roots of (31) merge into a real double root, i.e. $\lambda_{3}=\lambda_{4}=\alpha$. Also $\Delta_{g}=0$ and it can easily be shown that $\alpha^{2}$ is the simple zero of $g$, while $\lambda_{2} \alpha$ is the double one. Since $g(0)>0\left(\right.$ for $\left.c_{0} \neq-1\right)$ and the relation (35) still holds, we can conclude that $0<\alpha^{2}<1$ if and only if $\left(c_{0}, c_{1}\right) \in \operatorname{int} \mathcal{E}$. This implies immediately that, for $\left(c_{0}, c_{1}\right) \in \operatorname{int} \mathcal{E}$, one has $\alpha<1$. When $c_{0}=-1$ it follows that $c_{1}=\frac{\sqrt{2}}{4}$ and $\alpha=\frac{1}{4}$.

Case $3-\Delta_{f}>0$ We are left with the case where the eigenvalues $\lambda_{2}, \lambda_{3}$ and $\lambda_{4}$ are all real and distinct. Simple calculations show that for points $\left(c_{0}, c_{1}\right) \in \mathcal{E}$, with $c_{1}>0$, one has

$$
\Delta_{f}=\left(3+4 c_{0}\right)^{2}\left(5+4 c_{0}\right)^{2}\left(21+40 c_{0}+16 c_{0}^{2}\right)\left(37+68 c_{0}+32 c_{0}^{2}\right)^{2}<0,
$$

which leads easily to the conclusion that

$$
\left\{\left(c_{0}, c_{1}\right) \in \mathbb{R} \times \mathbb{R}^{+}: \Delta_{f}=0\right\} \cap \mathcal{E}=\emptyset \quad \text { and } \quad\left\{\left(c_{0}, c_{1}\right) \in \mathbb{R} \times \mathbb{R}^{+}: \Delta_{f}>0\right\} \subset \operatorname{int} \mathcal{E} .
$$




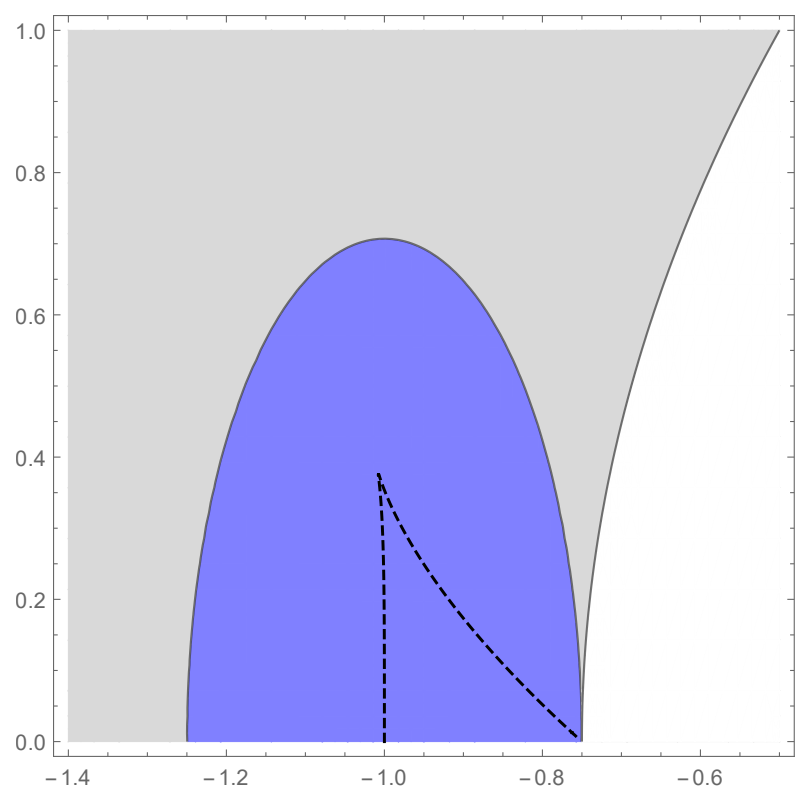

Figure 4: Stability (in the semi complex-plane $c_{0}+c_{1} \mathbf{i}, c_{1}>0$ ) of the set $\mathcal{P}_{8}$ of periodic points of period two: in the blue region, the set is attractive and in the gray region, the set is not attractive; in the white region, $\mathcal{P}_{8}$ is not defined; the dashed line corresponds to $\Delta_{f}=0$ (stability analysis not performed).

If $c_{1}=0$, then

$$
f(\lambda)=(\lambda-1)\left(\lambda-4 c_{0}-4\right)^{2},
$$

showing that the equation $f(\lambda)=0$ has the roots $\lambda=1$ and $\lambda=4\left(c_{0}+1\right)<1$. Assuming $c_{0} \neq-1$ and observing that $f(0) f(1)<0$, we can say, recalling that roots of a polynomial depend continuously on its coefficients, that for $0<c_{1} \ll 1$ (and $c_{0}$ such that $\Delta_{f}>0$ ) all the zeros of $f$ are in $(0,1)$. Using once more continuity arguments, it follows at once that, for any $\left(c_{0}, c_{1}\right)$ preserving $\Delta_{f}>0$, none of the zeros of $f$ can be greater than one, since $f(1) \neq 0$. Analyzing the function $f$ for $c_{0}=-1$ leads to the conclusion that also in this case, all the zeros of $f$ are in $(0,1)$.

We have just proved the first part of (ii). To complete the proof we need now to show that outside the ellipse $\mathcal{E}$ the eigenvalues have modulus greater than one. Observe that in such case, we have $\Delta_{f}<0$ (cf. (36)) and since $g(1)>0$ we conclude that the real root $\alpha^{2}+\beta^{2}$ (see Case 1 ) is greater than one and therefore $\left|\lambda_{3}\right|=\left|\lambda_{4}\right|>1$, as we wished to prove.

In what follows, we will assume that $\Delta_{f} \neq 0$, in which case the eigenvectors $\mathrm{v}_{1}, \mathrm{v}_{2}, \mathrm{v}_{3}, \mathrm{v}_{4}$ are linearly independent. ${ }^{5}$ It is simple to see that, due to the fact that $\mathrm{c}=c_{0}+c_{1} \mathbf{i}$, we have $\mathcal{O}(\mathrm{q}) \subseteq \operatorname{span}_{\mathbb{R}}\left(1, \mathbf{i}, \operatorname{vec}_{2} \mathrm{q}\right)$. If we analyze the expression of the eigenvectors (32), we conclude that the three eigenvectors $\mathrm{v}_{2}, \mathrm{v}_{3}, \mathrm{v}_{4}$ span

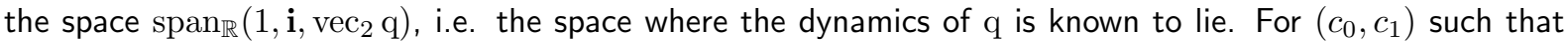
$\Delta_{f} \neq 0$ we thus have: for $\left(c_{0}, c_{1}\right)$ inside the ellipse $\mathcal{E}$ the set $\mathcal{P}_{8}$ is attractive and for values outside $\mathcal{E}$ (and satisfying $c_{1}^{2}>4 c_{0}+3$, which, we recall, is the condition for existence of $\mathcal{P}_{8}$ ) the set is not attractive; see Fig. 4.

Remark 5.4. We should note that the eigenvalues and eigenvectors of the Jacobian matrix of $f_{\mathrm{c}}^{2}$ at the only other point in $\mathcal{P}_{8}$ to which $\left(\left(f_{\mathrm{c}}^{2}\right)^{k}(\mathrm{q})\right)_{k}$ could eventually converge, i.e. the point $\tilde{\mathrm{q}}=-\frac{1}{2}+\frac{c_{1}}{2} \mathbf{i}-\alpha \operatorname{vec}_{2} \mathrm{q}$, are the same as the ones for the point $\mathrm{q}^{*}$; this shows that for values of $\left(c_{0}, c_{1}\right)$ outside the ellipse, we cannot obtain convergence for any point in $\mathcal{P}_{8}$.

Remark 5.5. It is worth mentioning that the eigenvector $\mathrm{v}_{1}$ associated with the eigenvalue equal to one gives the direction of the tangent space (line) to the circle $\mathcal{P}_{8}$ at $\mathrm{q}^{*}$.

\footnotetext{
${ }^{5}$ In the case where the two eigenvectors $\mathrm{v}_{3}$ and $\mathrm{v}_{4}$ have complex components, we can, as usual, replace them by the two vectors formed by the real and imaginary parts of one of them and the analysis that follow still holds.
} 
Remark 5.6. The previous analysis was done assuming that $c_{0}, c_{1}$ are such that $\Delta_{f} \neq 0$. When $\Delta_{f}=0$, we have a double eigenvalue $\lambda_{3}=\lambda_{4}$ which is defective. Hence, we do not have four independent eigenvectors $\mathrm{v}_{1}, \mathrm{v}_{2}, \mathrm{v}_{3}$ and $\mathrm{v}_{4}$, as before, but only three independent eigenvectors $\mathrm{v}_{1}, \mathrm{v}_{2}$ and $\mathrm{v}_{3}$, and thus our type of analysis cannot proceed.

\subsection{Stability of the set $\mathcal{P}_{9}$}

We now analyze the stability of the set

$$
\mathcal{P}_{9}=\left\{-\frac{1}{2}+q_{1} \mathbf{i}+\frac{c_{2}}{2} \mathbf{j}+q_{3} \mathbf{k}: q_{1}^{2}-q_{3}^{2}=\frac{c_{2}^{2}+4 c_{0}+3}{4}\right\} \backslash\left\{-\frac{1}{2}+\frac{c_{2}}{2} \mathbf{j}\right\},
$$

corresponding to the choice of a parameter $\mathrm{c}=c_{0}+c_{2} \mathbf{j}$, with $c_{2}>0$, but we exclude the case where $c_{2}^{2}+4 c_{0}+3=0$ (i.e. the case where the set consists of two straight lines). For $q=q_{0}+q_{1} \mathbf{i}+q_{2} \mathbf{j}+q_{3} \mathbf{k} \in \mathbb{H}_{\text {coq }}$, we use $\operatorname{vec}_{3} \mathrm{q}$ to denote the coquaternion $q_{1} \mathbf{i}+q_{3} \mathbf{k}$. Let q be a point chosen sufficiently close to the set $\mathcal{P}_{9}$ so that $\operatorname{det}\left(\operatorname{vec}_{3} \mathrm{q}\right)$ has the same sign as $c_{2}^{2}+4 c_{0}+3$ and let $\mathrm{q}^{*}$ be the point in $\mathcal{P}_{9}$ given by

$$
\mathrm{q}^{*}=-\frac{1}{2}+\frac{c_{2}}{2} \mathbf{j}+\alpha \operatorname{vec}_{3} \mathrm{q}, \text { with } \alpha=\sqrt{\frac{\frac{c_{2}^{2}+4 c_{0}+3}{4}}{\operatorname{det}\left(\operatorname{vec}_{3} \mathrm{q}\right)}} .
$$

It can be shown that one of the eigenvalues, $\lambda_{1}$, of the Jacobian matrix of $f_{\mathrm{c}}^{2}$ at $\mathrm{q}^{*}$ is equal to one and has associated eigenvector $\mathrm{v}_{1}=\left(0, q_{3}, 0, q_{1}\right)$. The other three eigenvalues $\lambda_{j} ; j=2,3,4$ of $\mathbf{J}_{2}\left(\mathrm{q}^{*}\right)$ are the roots of the cubic polynomial equation

$$
f(\lambda):=\lambda^{3}+A \lambda^{2}+B \lambda+C=0
$$

where

$$
A=-\left(4 c_{2}^{2}+8 c_{0}+9\right), \quad B=4\left(\left(c_{2}^{2}+2 c_{0}+2\right)^{2}+2\left(c_{0}+1\right)\right), \quad C=-16\left(c_{0}+1\right)^{2},
$$

and have associated eigenvectors given by

$$
\mathrm{v}_{i}=\left(C_{i}, q_{1}, D_{i}, q_{3}\right) ; i=2,3,4,
$$

where

$$
C_{i}=-\frac{1}{4 c_{2}^{2} \alpha}\left(-1+\lambda_{i}\right)\left(4+4 c_{0}+2 c_{2}^{2}-\lambda_{i}\right)
$$

and

$$
D_{i}=\frac{4+4 c_{0}+c_{2}^{2}-\lambda_{i}}{2 c_{2} \alpha} .
$$

We now prove that one of those eigenvalues always has modulus greater than one. First, observe that $f(\lambda)$ can be written as

$$
f(\lambda)=\lambda\left(\lambda-2 c_{2}^{2}-4 c_{0}-4\right)^{2}-\left(\lambda-4 c_{0}-4\right)^{2} .
$$

Since $f(1)=4 c_{2}^{2}\left(c_{2}^{2}+4 c_{0}+3\right)$, we are led to consider two cases:

Case $1-c_{2}^{2}+4 c_{0}+3<0$ In this case, we have $f(1)<0$ which means that $f(\lambda)=0$ has a root greater than one.

Case $2-c_{2}^{2}+4 c_{0}+3>0 \quad$ A direct calculation shows that the discriminant of $f$, given by

$$
\Delta_{f}=16 c_{2}^{4}\left(36+64 c_{0}^{3}+136 c_{2}^{2}+49 c_{2}^{4}+8 c_{2}^{6}+32 c_{0}^{2}\left(5+3 c_{2}^{2}\right)+4 c_{0}\left(33+58 c_{2}^{2}+12 c_{2}^{4}\right)\right),
$$

is positive, hence $f(\lambda)$ has three distinct real roots.

If $c_{2}=0, f(\lambda)=(\lambda-1)\left(\lambda-4 c_{0}-4\right)^{2}$, i.e. $\lambda_{2}=1$ and $\lambda_{3}=\lambda_{4}=4\left(1+c_{0}\right)>1$ are the roots of $f(\lambda)=0$. For $c_{2}$ sufficiently small, this equation has two real roots greater than one. Since $f(1) \neq 0$, this property holds in all region $c_{2}^{2}+4 c_{0}+3>0$. 
Now, as in the case of the analysis of the set $\mathcal{P}_{8}$, we assume that $\Delta_{f} \neq 0$, to guarantee that

the eigenvectors $\mathrm{v}_{1}, \mathrm{v}_{2}, \mathrm{v}_{3}, \mathrm{v}_{4}$ given by (38)-(39) are linearly independent. Here, we have that $\mathcal{O}(\mathrm{q}) \subseteq$ $\operatorname{span}_{\mathbb{R}}\left(1, \mathbf{j}, \operatorname{vec}_{3} q\right)$ and this set is spanned by the three eigenvectors $\mathrm{v}_{2}, \mathrm{v}_{3}$ and $\mathrm{v}_{4}$. Hence, for $\left(c_{0}, c_{2}\right)$ such that $\Delta_{f} \neq 0$ we may conclude that we do not have an attractive set.

Remark 5.7. We observe that the eigenvalues and eigenvectors of the Jacobian matrix of $f_{\mathrm{c}}^{2}$ at the only other point in $\mathcal{P}_{9}$ to which $\left(\left(f_{\mathrm{c}}^{2}\right)^{k}(\mathrm{q})\right)_{k}$ could eventually converge, i.e. the point $\tilde{\mathrm{q}}=-\frac{1}{2}+\frac{c_{2}}{2} \mathbf{j}-\alpha \operatorname{vec}_{3} \mathrm{q}$, are the same as the ones for the point $\mathrm{q}^{*}$, showing that we cannot obtain convergence for any point in $\mathcal{P}_{9}$.

Remark 5.8. We note that the vector $\mathrm{v}_{1}$ gives the direction of the tangent space (line) to the hyperbola $\mathcal{P}_{9}$ at $\mathrm{q}^{*}$.

We would like to refer that all the results concerning the stability discussed above were confirmed by numerical experiments. For each of the sets analyzed, several points in the set were selected and, at each of these points, random small perturbations were added. A large number of iterations were then performed and the final few iterates were recorded. In all the cases, the numerical results confirmed the behavior that we were expecting from the theory. For attractive sets, we always obtained convergence to a point in the set (more specifically, we observed convergence to the point $q^{*}$ referred to in the deduction of our results); for non-attractive sets, we either observed divergence (overflow in the computations) or convergence to a periodic point (sometimes of period greater than two) not in the set.

\subsection{Stability of the set $\mathcal{P}_{10}$}

We now consider the case of the set $\mathcal{P}_{10}$ given by (29), corresponding to the use of a parameter $\mathrm{c}=c_{0}+\mathbf{i}+\mathbf{j}$, $c_{0}<-\frac{3}{4}$. The eigenvalues of the Jacobian matrix $\mathbf{J}_{2}(\mathrm{q})$, for $\mathrm{q}=-1 / 2+q_{1} \mathbf{i}+q_{1} \mathbf{j}+q_{3} \mathbf{k}$ an arbitrary point in this set, are given by

$$
\lambda_{1}=\lambda_{2}=1, \quad \lambda_{3}=\lambda_{4}=4\left(1+c_{0}\right),
$$

with corresponding eigenvectors

$$
\mathrm{v}_{1}=(0,1,1,0), \mathrm{v}_{3}=\left(2 q_{3}\left(1-q_{1}\right), 0,0,1\right), \mathrm{v}_{4}=\left(2 q_{3}^{2}, 1,1,0\right) .
$$

We are now faced with a difficulty which did not occur before and which does not allow us to fully discuss the stability of the set: the double eigenvalue $\lambda_{1}=1$ is defective, with the corresponding eigenspace generated by a single vector $\mathrm{v}_{1}=(0,1,1,0)$. We know that, in this case, there is a generalized eigenvector associated with $\lambda_{1}=1$, i.e. a vector $\mathrm{w}_{2}$ of the form $\mathrm{w}_{2}=\left(-1 / 2, k, k+q_{3}^{2},-q_{1} q_{3}\right)$ satisfying

$$
\mathbf{J}_{2}(\mathrm{q}) \mathrm{w}_{2}=\mathrm{v}_{1}+\mathrm{w}_{2}
$$

and such that $\mathrm{v}_{1}, \mathrm{w}_{2}, \mathrm{v}_{3}$ and $\mathrm{v}_{4}$ are four independent vectors.

In this case, we numerically observed the following: when the initial point $\tilde{q}$ is a perturbation of the point $q$ of the form $\tilde{\mathrm{q}}=\mathrm{q}+\varepsilon_{1} \mathrm{v}_{1}+\varepsilon_{3} \mathrm{v}_{3}+\varepsilon_{4} \mathrm{v}_{4}$, the sequence of iterates converges to a point in the set $\mathcal{P}_{10}$ but, when $\tilde{\mathrm{q}}=\mathrm{q}+\varepsilon_{1} \mathrm{v}_{1}+\varepsilon_{2} \mathrm{w}_{2}+\varepsilon_{3} \mathrm{v}_{3}+\varepsilon_{4} \mathrm{v}_{4}$ with $\varepsilon_{2} \neq 0$, the behavior of the sequence of iterates is "unpredictable".

\section{Set Bifurcations}

In this section we draw the attention to some consequences of the results on the existence of fixed points and periodic points of period two, corresponding to values of the parameter $\mathrm{c}=c_{0} \in \mathbb{R}$, previously obtained. In what follows, $q_{0}^{(i)}$ and $q_{2}^{(i)} ; i=2,3,4,5$ are the points defined by (23).

For $c_{0}<-3 / 4$, consider an arbitrary point $\mathrm{q}=\frac{1}{2}+q_{1} \mathbf{i}+q_{2} \mathbf{j}+q_{3} \mathbf{k}$ in the set of fixed points $\mathcal{F}_{1}$ defined by (17) and let $\mathrm{q}^{(4)}$ and $\mathrm{q}^{(5)}$ be the two "neighboring" points defined by

$$
\begin{aligned}
& \mathrm{q}^{(4)}=q_{0}^{(4)}+q_{1} \mathbf{i}+\tilde{q}_{2} \mathbf{j}+q_{3} \mathbf{k}, \\
& \mathrm{q}^{(5)}=q_{0}^{(5)}+2 q_{0}^{(4)} q_{1} \mathbf{i}+2 q_{0}^{(4)} \tilde{q}_{2} \mathbf{j}+2 q_{0}^{(4)} q_{3} \mathbf{k},
\end{aligned}
$$




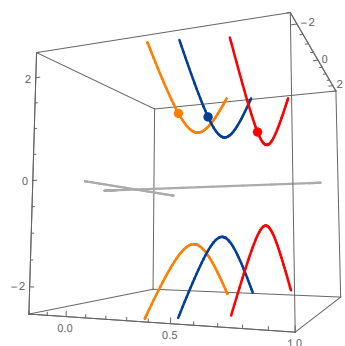

(a)

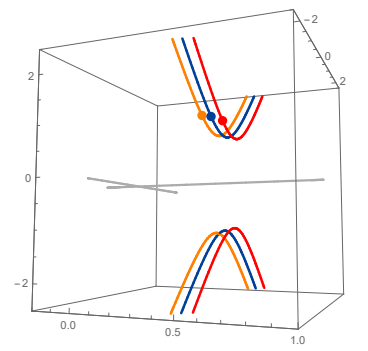

(b)

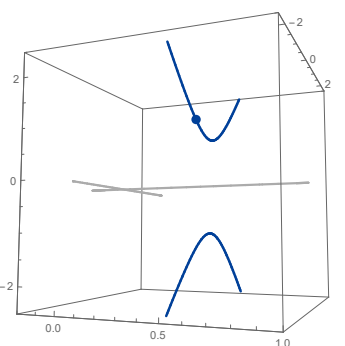

(c)

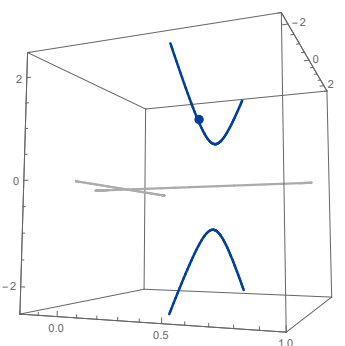

(d)

Figure 5: Plots, in the hyperplane $q_{3}=0$, of the set of fixed points $\mathcal{F}_{1}$ (in blue) and of the sets of periodic points of period two $\mathcal{P}_{4}$ (in orange) and $\mathcal{P}_{5}$ (in red) corresponding to parameters $c=c_{0} \in \mathbb{R}$, for different values of $c_{0}$; the real and imaginary axes are identified with gray lines; a particular point $q$ in $\mathcal{F}_{1}$ is identified with a blue point and the corresponding points $\mathrm{q}^{(4)}$ and $q^{(5)}$ defined by (40) with orange and red points, respectively; $(a) c_{0}=-0.9 ;(b) c_{0}=-0.76 ;(c) c_{0}=-0.75 ;(d) c_{0}=-0.6$. (For $c_{0} \geq-0.75$, the sets $\mathcal{P}_{4}$ and $\mathcal{P}_{5}$ are not defined.)

where $\tilde{q}_{2}$ is given by $\tilde{q}_{2}= \pm \sqrt{q_{1}^{2}-q_{3}^{2}+\left(q_{2}^{(4)}\right)^{2}}$ with the choice of the sign determined so that $\tilde{q}_{2}$ has the same sign as $q_{2} .{ }^{6}$ We have $\mathrm{q}^{(4)} \in \mathcal{P}_{4}$ and $\mathrm{q}^{(5)} \in \mathcal{P}_{5}$, hence $\mathrm{q}^{(4)}$ and $\mathrm{q}^{(5)}$ are periodic points of period two of $f_{c_{0}}$; furthermore, we also have that $f_{c_{0}}\left(\mathrm{q}^{(4)}\right)=\mathrm{q}^{(5)}$, which means that $\left\{\mathrm{q}^{(4)}, \mathrm{q}^{(5)}\right\}$ is a 2-cycle. Note that, as $c_{0}$ approaches the value $-3 / 4$ from the left, the two points $\mathrm{q}^{(4)}$ and $\mathrm{q}^{(5)}$ become closer to the fixed point $\mathrm{q}$. Hence, we can see this as a kind of local period-doubling bifurcation. Due to the arbitrariness in the choice of the point $\mathrm{q}$ in $\mathcal{F}_{1}$, we believe it makes sense to say that the family of coquaternionic quadratic maps $f_{\mathrm{c}}$ with $\mathrm{c}=c_{0} \in \mathbb{R}$ has a period-doubling bifurcation at $c_{0}=-3 / 4$ for the set of fixed points $\mathcal{F}_{1}$ and the two sets of periodic points of period two $\mathcal{P}_{4}$ and $\mathcal{P}_{5}$ : we call this a set bifurcation.

The above behavior is illustrated in Fig. 5 . In this figure we present plots, obtained by setting $q_{3}=0$, for different values of $c_{0}$, of the set of fixed points $\mathcal{F}_{1}$ (in blue) and of the sets of periodic points of period two $\mathcal{P}_{4}$ (in orange) and $\mathcal{P}_{5}$ (in red). (Recall that for $c_{0} \geq-3 / 4$, the sets $\mathcal{P}_{4}$ and $\mathcal{P}_{5}$ are not defined). A particular choice of $\mathrm{q}$ is displayed in the figure as a blue point and the corresponding points $\mathrm{q}^{(4)}$ and $\mathrm{q}^{(5)}$ are given as orange and red points, respectively.

For $\mathrm{c}=c_{0} \in \mathbb{R}$, let $\mathcal{P}$ be the set of fixed points of the map $f_{\mathrm{c}}^{2}$ given by

$$
\mathcal{P}=\left\{-\frac{1}{2}+q_{1} \mathbf{i}+q_{2} \mathbf{j}+q_{3} \mathbf{k}: q_{1}^{2}-q_{2}^{2}-q_{3}^{2}=c_{0}+\frac{3}{4}\right\} .
$$

Note that, for $c_{0}<-3 / 4, \mathcal{P} \equiv \mathcal{P}_{1}$, for $c_{0}=-3 / 4, \mathcal{P} \equiv \mathcal{P}_{6} \cup\{-1 / 2\}$ and for $c_{0}>-3 / 4, \mathcal{P} \equiv \mathcal{P}_{7}$, where $\mathcal{P}_{1}, \mathcal{P}_{6}$ and $\mathcal{P}_{7}$ are the sets of periodic points of period two of $f_{c_{0}}$ given by (22), (24) and (25), respectively. For $c_{0}<-3 / 4$, consider now an arbitrary point $\mathrm{q} \in \mathcal{P}$ and let $\mathrm{q}^{(2)}$ and $\mathrm{q}^{(3)}$ be the points defined in the following manner:

$$
\begin{aligned}
& \mathrm{q}^{(2)}=q_{0}^{(2)}+q_{1} \mathbf{i}+\tilde{q}_{2} \mathbf{j}+q_{3} \mathbf{k} \\
& \mathrm{q}^{(3)}=q_{0}^{(3)}+q_{1} \mathbf{i}+\hat{q}_{2} \mathbf{j}+q_{3} \mathbf{k}
\end{aligned}
$$

with $\tilde{q}_{2}$ and $\hat{q}_{2}$ given by $\tilde{q}_{2}= \pm \sqrt{q_{1}^{2}-q_{3}^{2}+\left(q_{2}^{(2)}\right)^{2}}, \hat{q}_{2}= \pm \sqrt{q_{1}^{2}-q_{3}^{2}+\left(q_{2}^{(3)}\right)^{2}}$, with the signs of $\tilde{q}_{2}$ and $\hat{q}_{2}$ chosen so that they have the same sign as $q_{2}$. We have that $\mathrm{q}^{(2)} \in \mathcal{P}_{2}$ and $\mathrm{q}^{(3)} \in \mathcal{P}_{3}$, hence $\mathrm{q}^{(2)}$ and $\mathrm{q}^{(3)}$ are both fixed points of the map $f_{c_{0}}^{2}$. Note that as $c_{0}$ approaches the value $-3 / 4$ from the left, the two points $\mathrm{q}^{(2)}$ and $\mathrm{q}^{(3)}$ become closer to the point $\mathrm{q}$. Observe also that, contrary to what happened in the previous case, $\mathrm{q}^{(2)}$ and $\mathrm{q}^{(3)}$ do not form a 2-cycle; hence, we can see this as a kind of local pitchfork bifurcation for

\footnotetext{
${ }^{6}$ One can show that the conditions $c_{0}<-3 / 4$ and $\mathrm{q} \in \mathcal{F}_{1}$ ensure that $q_{1}^{2}-q_{3}^{2}+\left(q_{2}^{(4)}\right)^{2}>0$.
} 

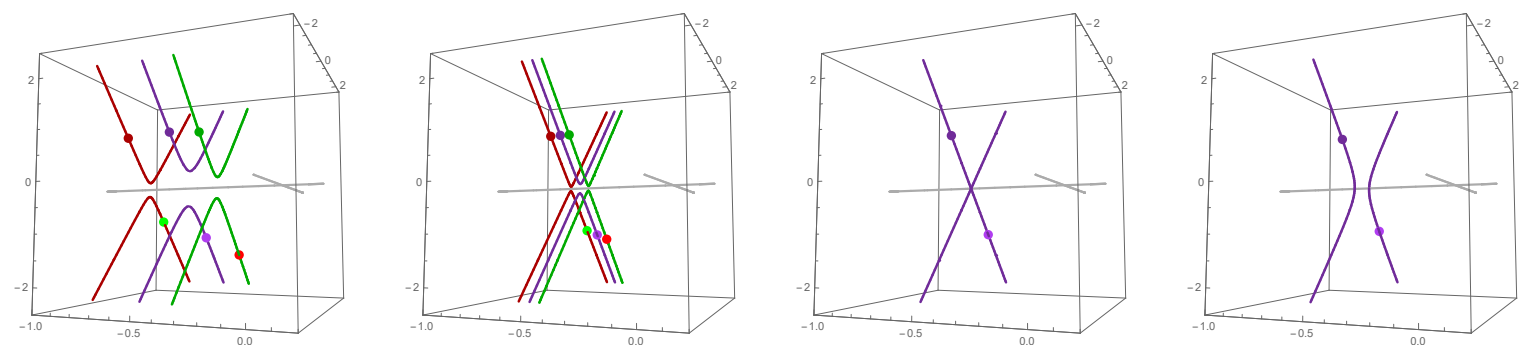

Figure 6: Plots, in the hyperplane $q_{3}=0$, of the sets of fixed points of the map $f_{c_{0}}^{2}, \mathcal{P}$ (in dark purple), $\mathcal{P}_{2}$ (in dark red) and $\mathcal{P}_{3}$ (in dark green), corresponding to parameters $\mathrm{c}=c_{0} \in \mathbb{R}$, for different values of $c_{0}$ (for $c_{0} \geq-0.75$, the sets $\mathcal{P}_{2}$ and $\mathcal{P}_{3}$ are not defined); the real and imaginary axes are identified with gray lines; a particular point $\mathrm{q}$ in $\mathcal{P}$ is identified with a dark purple point and the corresponding points $\mathrm{q}^{(2)}$ and $q^{(3)}$ defined by (41) with dark red and dark green points, respectively; the images of these points under the map $f_{c_{0}}$ are identified by using points with the same color, but in a lighter shade; $(a) c_{0}=-0.9 ;(b) c_{0}=-0.76$; (c) $c_{0}=-0.75 ;(d) c_{0}=-0.6$.

the map $f_{c_{0}}^{2}$ and again, due to the arbitrariness in the choice of $q \in \mathcal{P}$, it is natural to say that the family of coquaternionic maps $f_{\mathrm{c}}^{2}$ with $\mathrm{c}=c_{0} \in \mathbb{R}$ has a set pitchfork bifurcation at $c_{0}=-3 / 4$ for the set of points $\mathcal{P}$ and the two sets of points $\mathcal{P}_{2}$ and $\mathcal{P}_{3}$. Fig. 6 gives an illustration of this behavior. In this figure we present plots, obtained by setting $q_{3}=0$, for different values of $c_{0}$, of the sets $\mathcal{P}$ (in dark purple), $\mathcal{P}_{2}$ (in dark red) and $\mathcal{P}_{3}$ (in dark green). (For $c_{0} \geq-3 / 4$, the sets $\mathcal{P}_{2}$ and $\mathcal{P}_{3}$ are not defined). A particular choice of $q$ is indicated in the figure as a dark purple point and the corresponding points $\mathrm{q}^{(2)}$ and $\mathrm{q}^{(3)}$ are given as a dark red point and a dark green point, respectively. The images, under the map $f_{c_{0}}$, of these points are also displayed in the figure, using the same colors but in a lighter shade; these images illustrate the statement made above about $\left\{\mathrm{q}^{(2)}, \mathrm{q}^{(3)}\right\}$ not being a 2-cycle.

\section{Conclusions}

In the complex case, it is well-known that any arbitrary quadratic map is conjugate to a map of the form $f(z)=z^{2}+c$ and hence we need only to study this class of simple quadratics to understand the dynamics of all quadratic polynomials. The situation is, however very different in the coquaternionic case. Observe that, due to the non-commutativity of the product of coquaternions, a general quadratic coquaternionic polynomial is of the form

$$
\sum_{j=0}^{m} a_{j} x b_{j} x c_{j}+\sum_{k=0}^{n} d_{k} x e_{k}+c,
$$

and no conjugacy equivalence to a simpler form is known. In this paper, we considered only the simple family of coquaternionic quadratic maps of the form $f_{\mathrm{c}}(\mathrm{q})=\mathrm{q}^{2}+\mathrm{c}$, analogous to the one studied in the complex case, allowing us to establish some comparisons between the two settings. The fixed points and periodic points of period two of this family of maps were determined and a new feature of the coquaternionic dynamics was observed: the appearance of sets of non-isolated such points. This brought the need to consider a different concept of stability. The analysis of the stability - in this new sense - of the sets of fixed points and periodic points of period two was then performed. This analysis revealed another interesting aspect of the coquaternionic dynamics, not present in the complex case: the coexistence, for some choices of the parameter, of two distinct attractive 2-cycles, one, corresponding to the classical case, formed by two isolated points, and another which is a made up of non-isolated points.

Finally, for real values of the parameter c, we identified phenomena which can be seen as period-doubling and pitchfork bifurcations but applied to sets. The important differences from the complex setting, already observed in the simple case of fixed points and periodic points of period two of the quadratic family $f_{\mathrm{c}}(q)=$ $q^{2}+\mathrm{c}$, lead us to believe that the study of the dynamics of more general coquaternionic quadratic maps is an 
area of research worth exploring.

\section{Acknowledgments}

The authors would like to thank an anonymous referee for the careful reading of this manuscript and for the detailed and constructive suggestions.

Research at CMAT was financed by Portuguese funds through FCT - Fundação para a Ciência e a Tecnologia, within the Project UID/MAT/00013/2013. Research at NIPE has been carried out within the funding with COMPETE reference number POCl-01-0145-FEDER-006683, with the FCT/MEC's (Fundação para a Ciência e a Tecnologia, I.P.) financial support through national funding and by the ERDF through the Operational Programme on "Competitiveness and Internationalization - COMPETE 2020" under the PT2020 Partnership Agreement.

\section{References}

[1] Alagöz, Y., Oral, K. H. \& Yüce, S. [2012] "Split quaternion matrices," Miskolc Math. Notes 13, pp. 223232.

[2] Artzy, R. [1992] "Dynamics of quadratic functions in cycle planes," J. Geom. 44, pp. 36-32.

[3] Aulbach, B. [1984] Continuous and Discrete Dynamics near Manifolds of Equilibria, Lecture Notes in Mathematics, Vol. 1058, Springer-Verlag, Berlin.

[4] Baptista A. N., Correia Ramos, C. \& Martins, N. [2012] "Iteration of quadratic maps on matrix algebras," Int. J. Bifurcation and Chaos 22(6), pp. 1250150-1-1250250-7.

[5] Bedding, S \& Briggs, K. [1995] "Iteration of quaternion maps," Int. J. Bifurcation and Chaos 5(3), pp. 877-881.

[6] Bedding, S \& Briggs, K. [1996] "Iteration of quaternion functions," Amer. Math. Monthly 103, pp. 654664.

[7] Brenner, J. L. [1951] "Matrices of quaternions," Pacific J. Math 1(3), pp. 329-335.

[8] Buchanan, W., Gomatam, J. \& Steves, B. [2002] "Generalized Mandelbrot sets for meromorphic complex and quaternionic maps," Int. J. Bifurcation and Chaos 12(8), pp. 1755-1777.

[9] Cockle, J. [1849] "On systems of algebra involving more than one imaginary; and on equations of the fifth degree," Philosophical Magazine 35(3), pp. 434-435.

[10] Fauser, B. [1996] "Clifford algebraic remark on the Mandelbrot set of two-component number systems," Adv. Appl. Clifford Algebr. 6(1), pp. 1-26.

[11] Fishback, P. E. [2005] "Quadratic dynamics in binary number systems," J. Difference Equ. Appl. 11(7), pp. 597-603.

[12] Galeeva, R. \& Verjovsky, A. [1999] "Quaternion dynamics and fractals in $\mathbb{R}^{4}$," Int. J. Bifurcation and Chaos 9(9), pp. 1771-1775.

[13] Gomatam, J., Doyle, J., Steves, B. \& McFarlane, I. [1995] "Generalization of the Mandelbrot set: quaternionic quadratic maps," Chaos Solit. Fract. 5, pp. 971-986.

[14] Inoguchi, J. and Toda, M. [2004] "Timelike minimal surfaces via loop groups," Acta Appl. Math. 83(3), pp. 313-355.

[15] Kula, L. \& Yayli, Y. [2007] "Split quaternions and rotations in semi Euclidean space $E_{2}^{4}$," J. Korean Math. Soc. 44, pp. 1313-1327. 
[16] Nakane, S. [2005], "Dynamics of a family of quadratic maps in the quaternion space," Int. J. Bifurcation and Chaos 15(8), pp. 2535-2543.

[17] Senn, P. [1990] "The Mandelbrot set for binary numbers," Amer. J. Phys. 58(10), pp. 1018.

[18] Serenevy, A. K. [2001] "Dynamics of polynomial maps of $2 \times 2$ real matrices," In Proc. 16th Summer Conf. General Topology and Its Applications 2001/2002, pp. 763-778. 